\title{
TeV candidate BL Lac objects
}

\author{
L. Costamante ${ }^{1,2}$ and G. Ghisellini ${ }^{2}$
}

1 Università Statale di Milano, via Celoria 16, 20133 Milano, Italy

2 Osservatorio Astronomico di Brera, via Bianchi 46, 23807 Merate, Italy

Received 1 October 2001 / Accepted 6 December 2001

\begin{abstract}
The TeV emission of low power BL Lac objects has been established by the detection of an handful of them. The knowledge of the level of the TeV emission and its spectrum can shed light on the particle acceleration mechanisms, and it is especially important to assess the still uncertain level of the far infrared background radiation, which can absorb the $\mathrm{TeV}$ photons through photon-photon interactions. In view of these implications, it is necessary to enlarge the number of TeV detected sources, and to find them at different redshifts. To this aim, we propose a general and simple criterium to select the best $\mathrm{TeV}$ candidates, and produce a list of them with flux estimates above $40 \mathrm{GeV}, 300 \mathrm{GeV}$ and $1 \mathrm{TeV}$.
\end{abstract}

Key words. galaxies: jets - galaxies: nuclei - BL Lacertae objects: general - radio continuum: galaxies $\mathrm{X}$-rays: galaxies

\section{Introduction}

The discovery that blazars (Flat Spectrum Radio Quasars and BL Lac objects) are very strong $\gamma$-ray emitters have renewed the interest about them, and opened new perspectives in the comprehension of the physics of these objects.

The observations by EGRET (Hartmann et al. 1999), onboard the Compton Gamma Ray Observatory, led to the discovery that blazars emit most of their power in the $\gamma$-ray band, and that their Spectral Energy Distribution (SED) is characterized by two broad peaks, now commonly (but not unanimously), interpreted as due to synchrotron and inverse Compton (IC) radiation, respectively (e.g. Maraschi et al. 1992; Dermer et al. 1992; Sikora et al. 1994; Ghisellini \& Madau 1996; but see Mannheim 1993; Rachen 1999; Muecke \& Protheroe 2000; Aharonian 2000 for a different interpretation). For the first time we were able to study their entire SED in a comprehensive way, finding differences among subclasses of blazars about the frequency location of the two broad peaks, their relative luminosity and variability behaviors in different bands. Considering the EGRET sources and three complete blazar samples, it was found a correlation between the location of the two broad peaks and the observed bolometric luminosity (Fossati et al. 1998, hereafter F98; Ghisellini et al. 1998). Blazars seem to form a well defined sequence, with low powerful objects having both peaks at a similar level of luminosity, and located at higher frequencies than in more powerful objects, in which the IC peak dominates the emission. In the BL Lac

Send offprint requests to: L. Costamante,

e-mail: costa@merate.mi.astro.it class, the first kind of sources were named High frequency Peak BL Lacs (or HBL for short) by Padovani \& Giommi (1995), while the latter subclass was called Low frequency Peaked BL Lacs (LBL). Recent observations of high redshift $(z>4)$ blazars (Fabian et al. 2001a, 2001b; Celotti 2001), and of low power BL Lac objects (Costamante et al. 2001a, 2001b) have extended the blazar sequence at both ends, resulting in agreement with the original trend.

At $\mathrm{TeV}$ energies, the detection and study of blazar objects by ground based Cherenkov telescopes have been limited up to now to few HBL sources (Mkn 501, Mkn 421, PKS 2155-304, 1ES 2344+514, see Catanese \& Weekes 1999), though disclosing new and fundamental aspects of the blazar behavior. These observations monitor the behavior of the most energetic electrons of the source, thus shedding light on the acceleration mechanism working at the most extreme conditions. The very rapid variability observed at these energies (Mkn 421 doubled its TeV flux in less than 20 min, see Gaidos et al. 1996; Catanese \& Weekes 1999), coupled with the requirement that the source must be transparent with respect to the photonphoton process, tightly constrains the physical parameters, such as the source size and its beaming factor. In addition, Mkn 421 showed a tight correlation between the emission in the $\mathrm{X}$-ray and $\mathrm{TeV}$ bands (Maraschi et al. 1999; Takahashi et al. 2000; Krawczynski et al. 2001), implying that the radiation produced in the two bands is co-spatial and produced by the same electrons: this is of crucial importance to constrain any emission model.

The strong connection between the $\mathrm{TeV}$ and X-ray emission was also clearly evident during the 1997 flare of Mkn 501, when this source was observed by the X-ray 
satellite BeppoSAX in an extreme spectral state, with a synchrotron peak frequency close to $100 \mathrm{keV}$ or even more (Pian et al. 1998). Mkn 501 was found to have increased at least tenfold its luminosity, with most of it radiated at high X-ray energies. At the same time, the source underwent a major flare in the TeV band (Catanese et al. 1997b; Aharonian et al. 1997; Protheroe et al. 1997; DjannatiAtaï et al. 1999), and continued to be active (and well visible) in the TeV band for several months. This dramatic behavior can be explained by a synchrotron inverse Compton model, taking into account the effects introduced by the Klein Nishina scattering cross section and the constraints posed by the transparency of the source with respect to photon-photon collisions producing electron-positron pairs. It is not clear if the simultaneous variations in the $\mathrm{X}$-ray and $\mathrm{TeV}$ bands can be completely accounted for by a simple one-zone homogeneous synchrotron selfCompton model (see Tavecchio et al. 2001), or if we need some extra and more quiescent source of IR-optical photons (i.e. Ghisellini 1999).

$\mathrm{TeV}$ observations of BL Lacs are also particularly interesting because, being the only extragalactic sources known to emit at these energies, allow an independent estimate of the extragalactic IR background (IRB). Direct measurements of the IRB are affected (up to now) by relatively large uncertainties, due to the heavy contamination of foreground objects (for a review see Hauser \& Dwek 2001). Because TeV photons can be absorbed by IR photons for the pair production mechanism, the analysis/study of high energy spectra from sources at different redshifts gives an independent measure of the IRB level (Stecker et al. 1992).

These kind of studies are just started, and the first conclusions are based on the two most observed TeV BL Lacs, namely Mkn 421 and Mkn 501. The main uncertainty here is the knowledge of the primary blazar spectrum, which could have an intrinsic cut-off at high energies, or could be affected by absorption due to IR photons produced locally. A first and preliminary confirmation of the IRB absorption has been obtained comparing the spectra of Mkn 421 and Mkn 501, showing a cut-off in the power-law spectra at approximately the same energies (Krennrich et al. 2001), as expected since they have similar redshifts.

The direct measurements of the IRB flux lead to predict quite a strong absorption at $\mathrm{TeV}$ energies. If true, this in turn would imply an unusual primary spectrum above $\sim 1 \mathrm{TeV}$, which must have an excess above the extrapolation from lower energies, leading to the so-called IRB-TeV puzzle (see e.g. Protheroe \& Meyer 2000; Aharonian et al. 2001; Berezinsky 2001) ${ }^{1}$.

\footnotetext{
1 It has been proposed that this "puzzle" could be solved by quantum-gravity theories predicting the breaking of Lorentz invariance: as a consequence there should be a modification in the energy threshold for the $\gamma-\gamma \rightarrow \mathrm{e}^{ \pm}$process, explaining why $\mathrm{TeV}$ photons are not heavily absorbed by the IRB (AmelinoCamelia \& Piran 2001).
}

However, to draw unambiguous conclusions, we need an ensemble of sources located at different redshifts and a detailed knowledge of the $\mathrm{X}$-ray flux and spectrum: being produced by the same electrons (in synchrotron inverse Compton models), this would help in predicting the shape of the $\mathrm{TeV}$ emission.

To this aim we consider in this paper several published samples of bright BL Lac objects, for a total of 246 different objects, and propose a simple and handy tool to identify and select the most promising candidates. The main point we emphasize concerns the requirement of both high energy electrons and sufficient seed photons to originate the $\mathrm{TeV}$ emission. We therefore consider as best candidates those BL Lac objects having not only their synchrotron peak located at high energies, but also having sufficient radio-through-optical flux.

We therefore expand the work first done by Stecker et al. (1996), concerning only the Einstein Slew survey sample of BL Lac objects, both by considering other samples and also by introducing a different, albeit still simple, criterium to identify the best candidates.

\section{BL Lac objects detected at $\mathrm{TeV}$ energies}

At present, only few blazars have been detected by Cherenkov telescopes. In Table 1 we report the basic properties, fluxes and detection thresholds for those objects detected at least once above $5 \sigma$ (see Catanese \& Weekes 1999 and references therein). We have also added 1ES $1426+428$, whose detection was reported at about the $4.5 \sigma$ level by WHIPPLE (Horan D. et al. 2000). This was confirmed by CAT (Djannati-Atai, priv. comm.), by WHIPPLE again in 2001 (Horan D. et al. 2001), and by HEGRA (Aharonian 2001b). The latter two detections were at the $5 \sigma$ level.

Of these objects however, only Mkn 421 and Mkn 501 have been firmly confirmed by repeated detections by many Cherenkov telescopes, and since 1995 they have been extensively monitored. Thanks to their relatively high fluxes and repeated activity states, these are also the only two sources with spectral informations up to $\sim 17$ and $20 \mathrm{TeV}$ (Krennrich et al. 2001).

Two other objects have been reported to be $\mathrm{TeV}$ emitters, but with a somewhat lower significance. 1ES $1959+650$ was observed in 1998 by the UTAH Seven Telescope Array, and found with an excess of $3.9 \sigma$, reaching in two periods $\sim 5 \sigma$, but no fluxes were reported (Nishiyama et al. 1998). 3C 66A was instead detected once at $\sim 4.2 \sigma$ by the Crimean Astrophysical Observatory in 1996 (Neshpor et al. 1998), with a detection threshold at $0.9 \mathrm{TeV}$. If confirmed, this source could be extremely important, since due to the high redshift $(z=0.444)$, its TeV emission should be suppressed according to the actual estimates on the $\gamma-\gamma \rightarrow \mathrm{e}^{ \pm}$optical depth due to the IRB (Stecker \& De Jager 1998). 


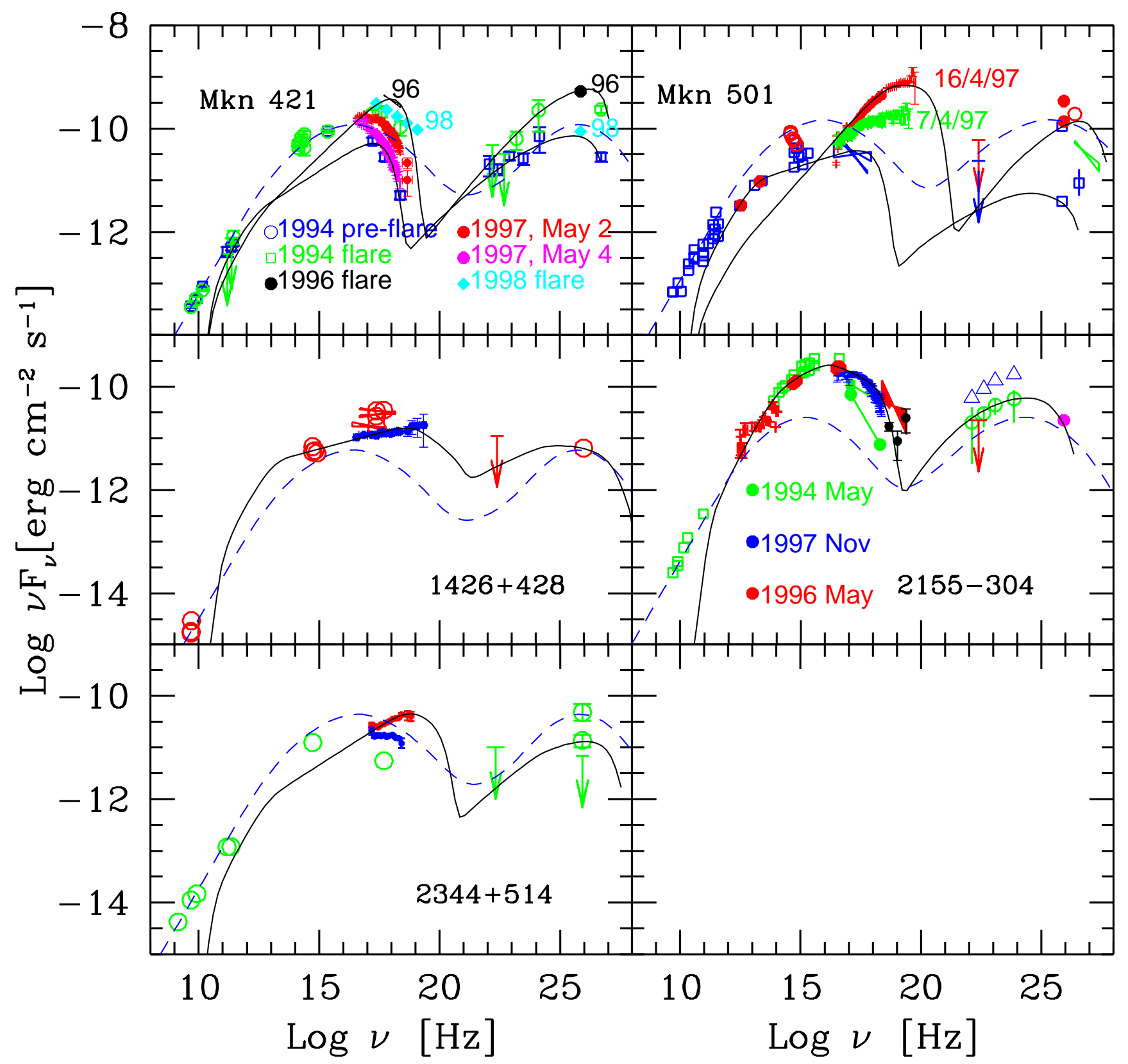

Fig. 1. Spectral energy distributions of the 5 BL Lac objects already detected at TeV energies. The solid and dashed lines refer to the SSC model described in Sect. 3.2 and to the SED constructed using the parameterization described in Fossati et al. (1998), with the modification described in this paper. The input parameters used for the SSC models are given in Ghisellini et al. (2001).

Table 1. Data for the BL Lac objects detected in the TeV band. Significances are listed only for unconfirmed sources. Data from Catanese \& Weekes (1999) and Krennrich et al. (2001), and references therein. 1) integrated flux in units of $10^{-11}$ photons $\mathrm{cm}^{-2} \mathrm{~s}^{-1}$.

\begin{tabular}{lllllllll}
\hline Source & $z$ & $\begin{array}{l}F_{5} \mathrm{GHz} \\
\mathrm{Jy}\end{array}$ & $\begin{array}{l}F_{5500 \mathrm{~A}} \\
\mathrm{mJy}\end{array}$ & $\begin{array}{l}F_{1 \mathrm{keV}} \\
\mu \mathrm{Jy}\end{array}$ & $F_{\mathrm{TeV}}^{1}$ & $\begin{array}{l}E_{\mathrm{th}} \\
\mathrm{TeV}\end{array}$ & Signif. & Tel. \\
\hline Mkn 421 & 0.031 & 0.722 & 9.75 & 9.98 & $1-80$ & 0.3 & conf. & WHIPPLE, CAT, HEGRA \\
1ES 1426+428 & 0.129 & 0.038 & 1.56 & 6.83 & 0.9 & 0.4 & $4.5-5 \sigma$ & WHIPPLE, (CAT), HEGRA \\
Mkn 501 & 0.034 & 1.371 & 10.31 & 9.44 & $0.8-49$ & 0.3 & conf. & WHIPPLE, CAT, HEGRA \\
PKS 2155-304 & 0.116 & 0.310 & 22.34 & 14.6 & 4.2 & 0.3 & $6.8 \sigma$ & Durham Mark6 \\
1ES 2344+514 & 0.044 & 0.215 & 3.54 & 2.91 & 1.7 & 0.35 & $5.2 \sigma$ & WHIPPLE \\
\hline
\end{tabular}




\section{The Synchrotron self-Compton process and $\mathrm{TeV}$ emission}

The BL Lac objects so far detected at TeV energies are relatively low powerful blazars, with no broad emission lines and no signs of thermal emission (i.e. the blue bump) produced by the accretion disk. This suggests and favors a synchrotron self-Compton (SSC) origin of the TeV flux. However this may be an over-simplification, since, besides the jet region containing the energetic electrons responsible for the high energy emission, other sites, both in the jet and externally to it (e.g. a molecular torus, a thin scattering plasma surrounding the jet, or the walls of the jet itself), may be important in producing the soft seed photons to be scattered at high energies. However, a simple one-zone and homogeneous SSC model can account for the observed SED of all the TeV BL Lacs, and due to its simplicity it is the basic framework we will use in the following. It is also the model assumed by the previous work on this issue, namely the paper by Stecker et al. (1996), which we discuss below, before introducing our views.

\subsection{The Stecker et al. (1996) scenario}

Based on the SSC framework, Stecker et al. (1996) have used simple scaling arguments to predict the $\gamma$-ray fluxes for HBL objects, and so to select good candidates for $\mathrm{TeV}$ emission. According to the SSC model, the inverse Compton component has a spectrum which is similar to the synchrotron one (both roughly parabolic on a logarithmic $\nu F_{\nu}$ plot, Macomb et al. 1995, F98), but upshifted by $\sim \gamma_{\text {peak }}^{2}$ (in the Thomson regime), where $\gamma_{\text {peak }}$ is the Lorentz factor of the electrons emitting at the peak. Using as "template" the SED of Mkn 421, at the time the only HBL source detected both at GeV (EGRET) and TeV (WHIPPLE) energies (i.e. with informations on both sides of the Compton peak), they found an upshifting factor of $\sim 10^{9}$, and a $L_{\mathrm{C}} / L_{\text {syn }} \sim 1$. Assuming then for simplicity that all HBL objects have the same properties as those found for Mkn 421, the found upshifting factor allowed to derive the following scaling law:

$\frac{\nu_{\mathrm{o}} F_{\mathrm{o}}}{L_{\mathrm{syn}}} \simeq \frac{\nu_{\mathrm{GeV}} F_{\mathrm{GeV}}}{L_{\mathrm{C}}}$ and $\frac{\nu_{x} F_{x}}{L_{\mathrm{syn}}} \simeq \frac{\nu_{\mathrm{TeV}} F_{\mathrm{TeV}}}{L_{\mathrm{C}}}$,

where $F_{\mathrm{o}}$ is the monochromatic flux at the optical frequency $\nu_{\mathrm{o}}$. Given $L_{\mathrm{C}} / L_{\mathrm{syn}} \sim 1$, a direct relation for the energy fluxes is obtained, in the $\mathrm{GeV}$ and $\mathrm{TeV}$ ranges:

$\nu_{\mathrm{GeV}} F_{\mathrm{GeV}} \sim \nu_{\mathrm{o}} F_{\mathrm{o}}$ and $\nu_{\mathrm{TeV}} F_{\mathrm{TeV}} \sim \nu_{x} F_{x}$.

Quantitative estimates on the integral fluxes were then made using the average spectral index for BL Lacs between 0.1 and $10 \mathrm{GeV}(\alpha=0.8)$, and the Mkn 421 slope above $0.3 \mathrm{TeV}(\alpha=1.2)$.

In this scenario, therefore, the main selection criterium is the $\mathrm{X}$-ray flux level: the energy flux around $1-2 \mathrm{keV}$ gives directly the expected level of the emission roughly around $0.2-0.3 \mathrm{TeV}$. In this context, sources with the highest synchrotron $\nu_{\text {peak }}$ are expected to emit more in the

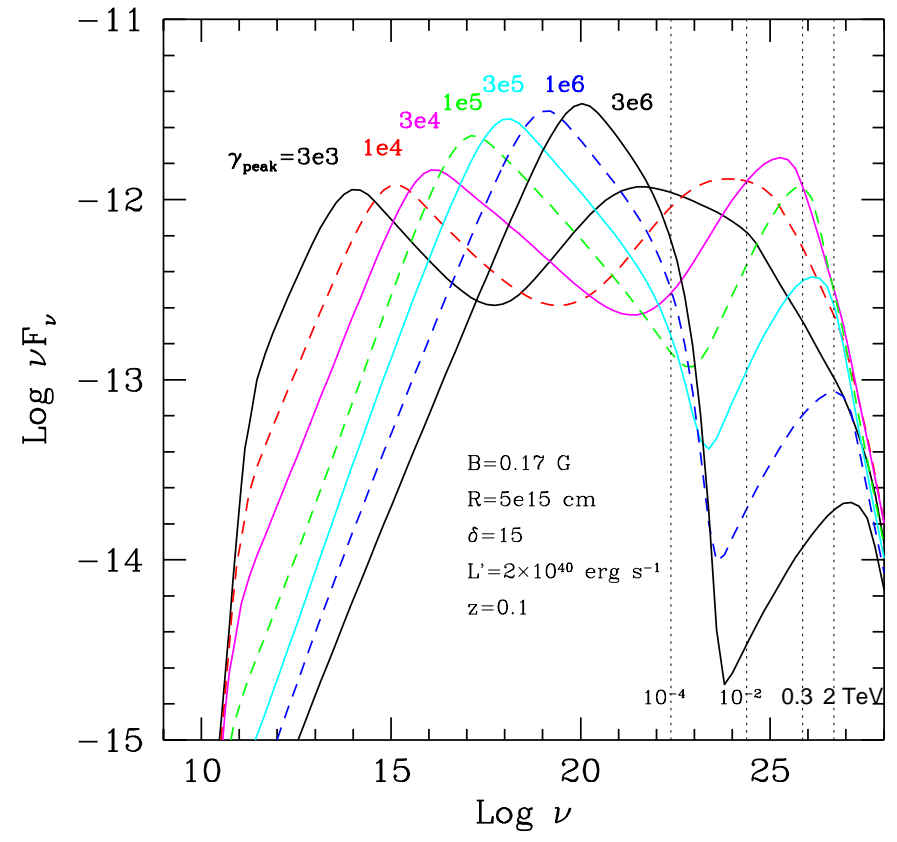

Fig. 2. SEDs calculated with a simple one-zone SSC model as described in Sect. 3.2, in which $\gamma_{\text {peak }}$ increases (as labeled). All other parameters are kept constant.

$\mathrm{TeV}$ band, since the Compton peak progressively moves into the $\mathrm{TeV}$ window.

\subsection{Energetic electrons and seed photons}

The two ingredients for the formation of a strong $\mathrm{TeV}$ emission by the synchrotron self Compton process are the density of electrons energetic enough to produce $\mathrm{TeV}$ photons and the density of seed photons to be scattered. For not too large synchrotron peak frequencies, the scattering process between photons at the synchrotron peak and the electron producing the peak itself is in the Thomson regime. In this case the relevant electrons can scatter the bulk of the synchrotron photons to high energies.

For larger synchrotron peak frequencies (and assuming the same value of the magnetic field) we have more energetics electrons in principle capable to produce photons of larger energies. However in this case the KleinNishina decline of the scattering cross section is important, disfavoring those scatterings between the relevant electrons and the synchrotron photons lying at the peak. In this case the scattering process producing $\mathrm{TeV}$ photons is produced by seed photons of lower energies, with a corresponding reduced synchrotron energy density. In other words, not all the synchrotron photons are used to form the Compton spectrum, but only the photons of energy (in the comoving, primed, frame) $h \nu^{\prime}<m_{\mathrm{e}} c^{2} / \gamma$ can efficiently (i.e. they scatter in the Thomson regime) contribute to the Compton emission. There is then a trade-off: to produce large $\mathrm{TeV}$ fluxes we need very energetic electrons, corresponding to very large $\nu_{\text {peak }}$, but we also need enough seed photons, and this requires not extreme values of $\nu_{\text {peak }}$. 


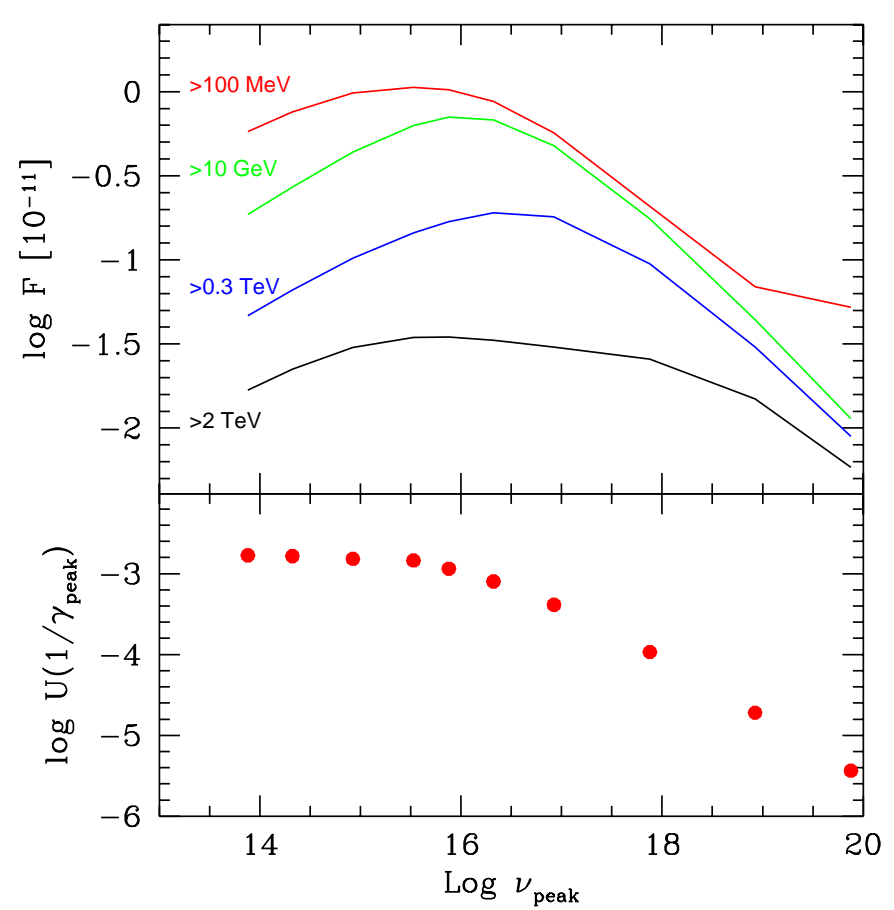

Fig. 3. Top panel shows the flux integrated above the indicated energies corresponding to the spectra of Fig. 2. $\nu_{\text {peak }}$ is the synchrotron peak frequency of the SEDs in Fig. 2. The bottom panel shows the radiation energy density available for scattering in the Thomson regime with electrons of energy $\gamma_{\text {peak }} m_{\mathrm{e}} c^{2}$.

To better illustrate this case we show in Fig. 2 a sequence of spectra derived by a standard SSC model in which all parameters but $\gamma_{\text {peak }}$ are kept constant. In this model it is assumed that relativistic electrons between $\gamma_{1}$ and $\gamma_{2}$ are injected at a constant rate $Q(\gamma) \propto$ $\gamma^{-2.5}\left[\mathrm{~cm}^{-3} \mathrm{~s}^{-1}\right]$, throughout a spherical source of radius $R$, magnetic field $B$ and beaming factor $\delta$. The steady state particle distribution is found through the continuity equation, accounting for synchrotron and inverse Compton radiative losses, electron positron pair production and Klein Nishina effects. Particles are assumed not to escape the source. In this case $\gamma_{\text {peak }}$ is always coincident with $\gamma_{1}$ (see Ghisellini et al. 1998 for more details of the model).

In Fig. 3 we report the flux integrated above a given frequency as a function of $\nu_{\text {peak }}$, corresponding to the spectra shown in Fig. 2. In the bottom panel we also show the comoving radiation energy density which is available for scattering in the Thomson limit. From both Figs. 2 and 3 we can see that the level of the TeV emission initially increases for increasing $\gamma_{\text {peak }}$, since in this case the scattering of the bulk of the synchrotron photons occurs in the Thomson regime, and the increase in $\gamma_{\text {peak }}$ results in more photons reaching $\mathrm{TeV}$ energies. This trend is reversed when $h \nu_{\text {peak }}^{\prime} \geq m_{\mathrm{e}} c^{2} / \gamma_{\text {peak }}$, e.g. when

$\nu_{\text {peak }} \geq 3.8 \times 10^{15} B^{1 / 3} \frac{\delta}{1+z} \mathrm{~Hz}$

where $\nu_{\text {peak }}=(4.3) \nu_{\mathrm{L}} \gamma_{\text {peak }}^{2} \delta /(1+z)$, and $\nu_{\mathrm{L}}=$ $e B /\left(2 \pi m_{\mathrm{e}} c\right)$ is the Larmor frequency. The increase in the synchrotron luminosity for increasing $\nu_{\text {peak }}$ is due to the corresponding decrease in the self Compton component: since all the injected power is assumed to be radiated, the sum of the synchrotron and the self Compton luminosities must be constant.

We conclude that very extreme BL Lacs, with very large values of $\nu_{\text {peak }}$, are not necessarily the best candidates to be strong $\mathrm{TeV}$ emitters since they can scatter relatively fewer photons. The best TeV BL Lac candidates should be the one with both a large $\nu_{\text {peak }}$ and a sufficiently strong soft seed IR photon emission.

\section{TeV candidate BL Lacs}

\subsection{The samples}

In order to select new candidates for $\mathrm{TeV}$ emission, we have assembled a catalogue of BL Lac objects using several published BL Lac samples, for which informations in all the three energy bands (radio, optical and X-ray) were available. We considered the Slew Survey Sample (Perlman et al. 1996), the Einstein Medium Sensitivity Survey (EMSS, Rector et al. 2000), the ROSAT All Sky Survey BL Lac sample (RASS, Bade et al. 1998), the ROSAT All Sky Survey - Green Bank sample (RGB, Laurent-Muehleisen et al. 1999), the EXOSAT archive BL Lac catalogue (Giommi et al. 1990; Sambruna et al. 1994) and the 1 Jy BL Lac sample (Stickel et al. 1993; Urry et al. 1996). We have also added all the HBL objects in Donato et al. (2001), who gives a list of all known blazars detected in $\mathrm{X}$-rays for which a measure of the spectral index was available.

The sources listed in more than one sample have been considered only once, and attributed with the following order: Slew, EMSS, RASS, RGB, EXOSAT, 1 Jy samples and those in the Donato et al. compilation (i.e. the EMSS sources in Figs. 4 and 5 are those not already in the Slew sample, the RASS sources are those not included in the EMSS and Slew samples, and so on). In this way we obtained an ensemble of 246 different objects. The flux informations used are those reported in the respective catalogue papers (see each reference). Because the data were often provided in different formats, we have uniformed all the fluxes to monochromatic fluxes at $5 \mathrm{GHz}, 5500 \AA$ and $1 \mathrm{keV}$, using the same spectral information adopted in the compilation of each sample, for consistency. In the radio band, the RASS sample reported the fluxes at $1.4 \mathrm{GHz}$ along with the radio spectral index, so we have calculated the flux at $5 \mathrm{GHz}$ assuming a power-law spectrum and the reported $\alpha_{\mathrm{R}}$. In the $\mathrm{X}$-ray band we calculated the fluxes at $1 \mathrm{keV}$ from integrated fluxes (RASS and RGB) or fluxes at $2 \mathrm{keV}$ (Slew, EMSS) using the $\mathrm{X}$-ray spectral indices there reported. The optical fluxes were calculated from the tabulated magnitudes, dereddened with the $A_{\mathrm{B}}$ values obtained from the NED database (Burstein \& Heiles 1982). When the optical flux was not at $5500 \AA$ ( $V$ filter $)$, we obtained it extrapolating from the flux at the different effective wavelength, assuming a power law spectrum 
of slope $\alpha_{\mathrm{opt}}=1$. The NED database was also used to check the redshift for all sources, 65 of which do not have a reported value.

For Figs. 4 and 5, the fluxes have been $\mathrm{K}$-corrected with the respective catalogue spectral indices, assuming power-law spectra. When the slope was not reported, we used $\alpha_{\mathrm{R}}=0$ and $\alpha_{\mathrm{opt}}=1$ for the $\mathrm{K}$-correction of the radio and optical fluxes, respectively. The $\mathrm{X}$-ray flux was $\mathrm{K}$-corrected using the reported spectral indices. For the $\mathrm{K}$-correction in case of unknown redshift we used $z=0.2$.

\subsection{Selection of TeV candidate BL Lacs}

Figure 4 shows the $\mathrm{X}$-ray flux as a function of the radio flux for the BL Lacs objects in our ensemble. Note the locations of the already $\mathrm{TeV}$-detected sources: they are among the brightest sources in both bands. This is not so obvious as it may seem at first sight, since a large X-ray to radio flux (hence, a lower radio emission for a given $\mathrm{X}$-ray flux) indicates a large synchrotron peak frequency (see e.g. Fig. 8 in F98), which is the first requirement to emit in the $\mathrm{TeV}$ band (we must have many electrons energetic enough to emit copiously at $\mathrm{TeV}$ energies). Consider also that the radio emission (at $5 \mathrm{GHz}$ ) must be produced in a large region of the jet (not to be self-absorbed), much larger than the part of the jet emitting high frequency radiation (as required by the very rapid variability). Therefore the link between the radio/X-ray emission and the $\mathrm{TeV}$ flux is more subtle.

We have interpreted this property in the following way: to produce a large $\mathrm{TeV}$ flux by the IC process we need many electrons of random Lorentz factors $\gamma \sim 10^{5}$ $10^{6}$. These electrons emit synchrotron photons of energies $h \nu=1.5 B\left(\gamma / 10^{5}\right)^{2}(\delta / 10) \mathrm{keV}$, with $B$ in Gauss. The seed photons most effective to interact with these electrons to produce $\mathrm{TeV}$ photons by the IC process are in the IRoptical band, since photons of higher frequencies scatter in the Klein Nishina regime. We therefore propose that the radio flux measures the level of the relevant seed photons. If this is the case, then, for a given X-ray flux, sources that are brighter in the radio band are more likely to be $\mathrm{TeV}$ emitters. And indeed the objects already detected in the $\mathrm{TeV}$ band are bright both in the radio and in the $\mathrm{X}$-ray bands. We think this is exactly the effect of the "trade-off" between high $\gamma_{\text {peak }}$ and the energy density of seed photons (see Sect. 3.2), since the objects with higher $\nu_{\text {peak }}$ are, on average, those with fainter radio emission for a given $\mathrm{X}$-ray flux, i.e. those with lower $\alpha_{\mathrm{rx}}$ (see the correlation between $\alpha_{\mathrm{rx}}$ and $\nu_{\text {peak }}$ in F98, Wolter et al. 1998; Costamante et al. 2001).

As shown in Fig. 4, in the region of high radio and $\mathrm{X}$-ray flux around the detected $\mathrm{TeV}$ objects there are also other sources, that we therefore consider good candidates for $\mathrm{TeV}$ emission. The extension of such region is, to some extent, subjective: the "rectangle" in Fig. 4 was drawn in order to include the already $\mathrm{TeV}$ detected sources and sources like Mkn 501 and Mkn 421 if they were at a redshift $\sim 0.1$. In Fig. 4 an increase of the redshift corresponds to decrease the fluxes along the lines of constant $\alpha_{\mathrm{rx}}$ (the changing $\mathrm{K}$-correction causes a negligible deviation, on this scale).

Figure 5 shows how the BL Lac objects are placed in the optical - X-ray flux plane. As the seed photons most effective for the $\mathrm{TeV}$ emission are in the IR-optical band, the optical flux could be in principle a better indicator of the density of seed photons than the radio flux. However, due to the possible contamination (either as emission and absorption) from the host galaxy (and uncertainties on the intervening reddening medium), we consider the radio flux a more reliable indicator of the low-energy nonthermal nuclear emission from these objects. Note that, in any case, all but one sources within the radio- $\mathrm{X}$-ray rectangle are also within the optical-X-ray one. For a given $\mathrm{X}$-ray flux, then, a relatively high emission in both the radio and optical bands should represent a reliable indication of a large energy density of seed photons.

Table 2 reports the list of the objects which are inside the two "rectangles" in Figs. 4 and 5, according to the values reported in the respective samples (see Sect. 4.1), and that therefore we consider the best candidates for a possible $\mathrm{TeV}$ detection.

\section{Prediction of the TeV flux}

To better quantify the predicted high energy flux of our best candidates we have collected from the literature other data for all the objects listed in Table 2, in order to construct their SED. We have then used two different methods to estimate their high energy emission. First, for each source, we have applied a SSC model, as explained below, aimed to fit the synchrotron component of their SED and to predict the inverse Compton spectrum. Then we have also calculated the predicted spectrum according to a slightly modified version of the parameterization given by F98, thought to describe the average SED of blazars.

Note that the predicted SEDs shown in Fig. 6 and the $\mathrm{TeV}$ fluxes listed in Table 3 do not take into account the absorption of $\mathrm{TeV}$ photons by the IRB, since this is indeed one of the important unknowns we would like to measure.

\subsection{Homogeneous Synchrotron self-Compton model}

We have applied a homogeneous, one-zone synchrotron self-Compton model to our best $\mathrm{TeV}$ candidate BL Lacs. This model is "one-zone version" of the model in Spada et al. (2001) and is described in detail in Ghisellini et al. (2001). The main characterizing feature of this model is the assumption of an particle injection mechanism lasting for a finite time. In this case the emitting particle distribution never reaches a complete steady state. A physical scenario where such a behavior naturally occurs is that of internal shocks (see e.g. Piran 1999; Ghisellini 1999; Spada et al. 2001), i.e. collisions of different parts of the jet plasma moving at slightly different speeds, naturally leading to shocks lasting for a finite time. This scenario 


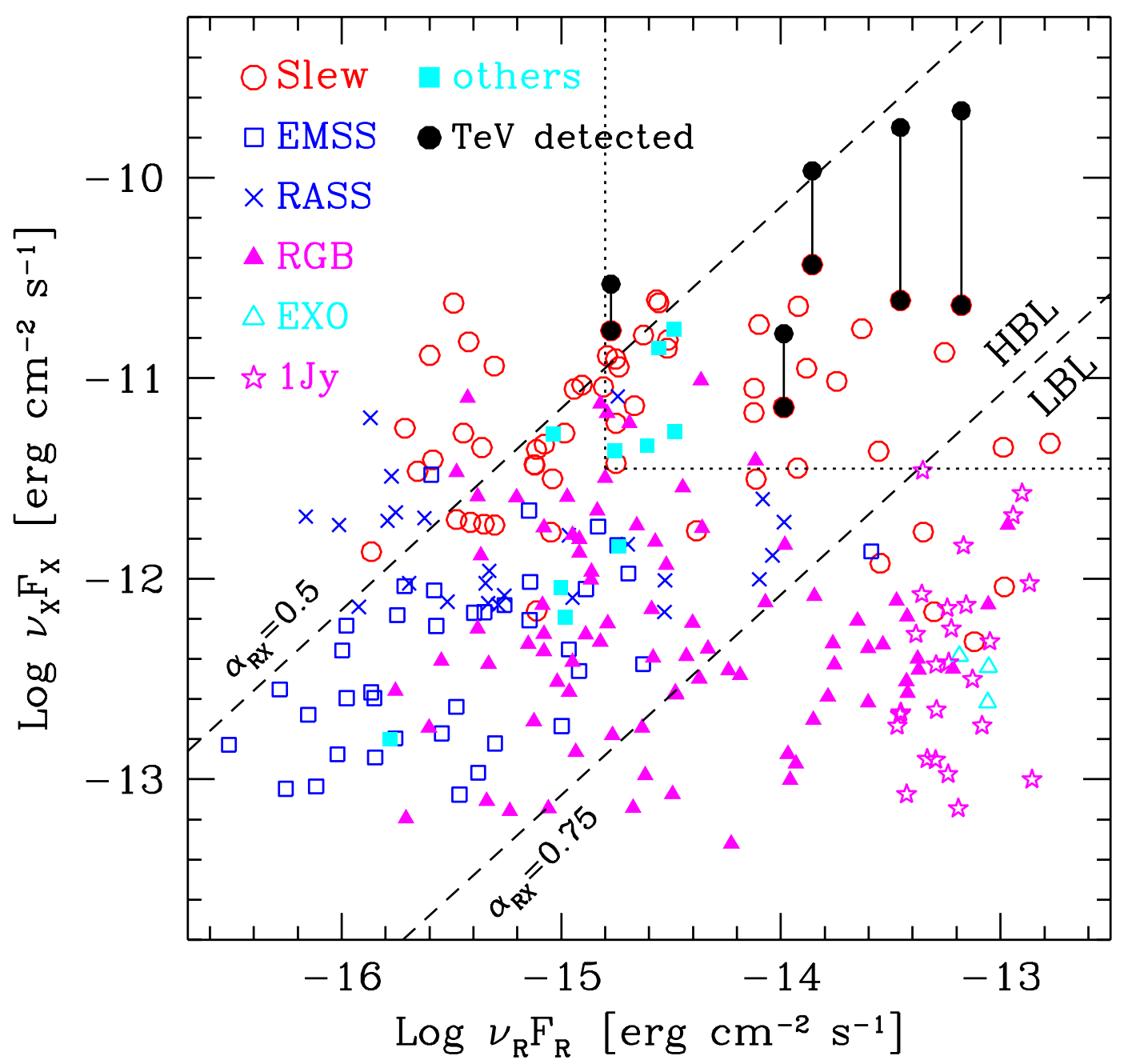

Fig. 4. BL Lac objects in the radio $(5 \mathrm{GHz})$ and $\mathrm{X}$-ray $(1 \mathrm{keV}) \nu F(\nu)$ plane. Sources belonging to different samples have different symbols, as labeled. The objects marked with filled circles are those already detected at TeV energies (from left to right, 1ES 1426+428, 1ES 2344+514, PKS 2155-304, Mkn 421, Mkn 501). Note that for these sources we have plotted two different X-ray states, connected by the vertical segment. The dotted lines delimiting the rectangle are at $F_{\mathrm{x}}=1.46 \mu \mathrm{Jy}$ and $F_{\mathrm{R}}=31.6 \mathrm{mJy}$.

is suggested by the rapid variability always present in BL Lac objects in general and HBL in particular, especially at high energies. We briefly outline here the other main assumptions of the model.

We assume that the jet is conical, with half opening angle $\psi \simeq 1 / \Gamma$, and approximate the emitting region as a cylinder, of radius $R(=\psi z$, where $z$ is the distance along the jet axis) and width $\Delta R^{\prime}=R / \Gamma$ (in the comoving frame, here $\Gamma$ is the bulk Lorentz factor). This corresponds to assume $\Delta R^{\prime}$ constant up to the first collision, in the internal shocks scenario (Spada et al. 2001). We derive the particle distribution assuming that the acceleration mechanism is in the form of a continuous injection of relativistic particles distributed in energy as a power law of index $n-1$ between $\gamma_{1}$ and $\gamma_{2}$. The injection is assumed to last for a finite time, set equal to $t_{\text {inj }}=\Delta R^{\prime} / c$. We define $\gamma_{\mathrm{c}}$ as the energy of those electrons that can cool in the injection time $t_{\text {inj }}$, i.e. $t_{\text {cool }}\left(\gamma_{c}\right)=t_{\text {inj }}$. At energies greater than $\gamma_{c}$, particles radiatively cool, and the distribution reaches a steady state in a time smaller than $t_{\mathrm{inj}}$. As a consequence, the emitting particle distribution is assumed to be a power law of index $n$ above $\gamma_{c}$. Below this value, there can be different cases according if $\gamma_{\mathrm{c}}$ is greater or smaller than $\gamma_{1}$. If $\gamma_{\mathrm{c}}>\gamma_{1}$, we have $N(\gamma) \propto \gamma^{-(n-1)}$ between $\gamma_{1}$ and $\gamma_{\mathrm{c}}$. Alternatively, if $\gamma_{\mathrm{c}}<\gamma_{1}$, then $N(\gamma) \propto \gamma^{-2}$ between $\gamma_{\mathrm{c}}$ and $\gamma_{1}$. We further assume that, below the minimum between $\gamma_{1}$ and $\gamma_{\mathrm{c}}, N(\gamma) \propto \gamma^{-1}$. According to these assumptions, the random Lorentz factor $\gamma_{\text {peak }}$ of the electrons emitting most of the radiation (i.e. emitting at the peaks of the SEDs) is determined by the importance of radiative losses and can have values within the range $\gamma_{1}-\gamma_{2}$. Its value is listed in Table 3. The source is assumed to emit an intrinsic luminosity $L^{\prime}$ and is assumed to be observed with the viewing angle $\theta$. All these input parameters are listed in Table 3, together with the predicted high energy photon fluxes above three representative frequencies. The resulting fits are shown in Fig. 6 as solid lines. 


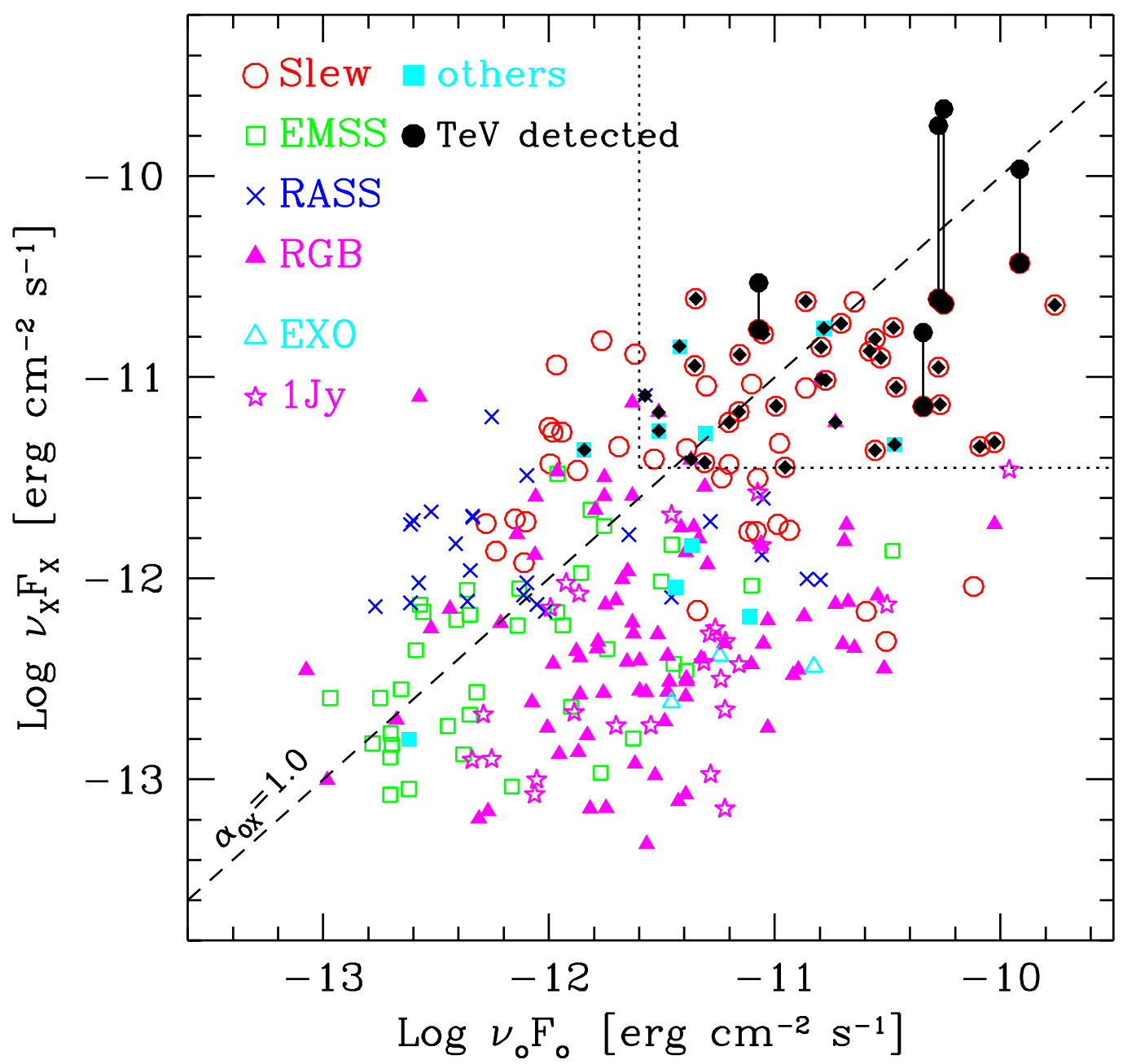

Fig. 5. BL Lac objects in the optical $(5500 \AA)$ and $\mathrm{X}$-ray $(1 \mathrm{keV}) \nu F(\nu)$ plane. Sources belonging to different samples have different symbols, as labeled. The objects marked with filled circles are those already detected at TeV energies (from left to right, 1ES 1426+428, 1ES 2344+514, Mkn 421, Mkn 501 and PKS 2155-304). Note that for these sources we have plotted two different X-ray states, connected by the vertical segment. The dotted lines delimiting the rectangle are at $F_{\mathrm{x}}=1.46 \mu \mathrm{Jy}$ and $F_{\mathrm{o}}=0.49 \mathrm{mJy}$. The objects marked also with a black diamond are those inside the rectangle of the $\nu_{\mathrm{x}} F_{\mathrm{x}}-\nu_{\mathrm{R}} F_{\mathrm{R}}$ plot (Fig. 4).

In conventional SSC models (Tavecchio et al. 1998), the knowledge of the location of the two peaks and their fluxes, together with the variability timescale, suffices to derive unambiguously all relevant physical parameters. Here, instead, we lack two important observables, namely the frequency and flux of the Compton peak. We therefore need to supply two additional relations with respect to the "standard" SSC model.

One of these relations comes from our assumption of finite injection time, resulting in a relation between $\nu_{\text {peak }}$ and the magnetic field. In fact, if synchrotron losses are relevant (as in the sources already detected at $\mathrm{TeV}$ energies, for which the high energy component never exceeds the synchrotron one), we have a constraint on the value of the magnetic field, controlling the radiative losses timescales and hence the value of $\gamma_{\text {peak }}$.

For the second unknown to be provided, we decided to limit the value of the beaming Doppler factor $\delta$ within the relatively narrow range $9<\delta<20$.
Note, however, that also within the previous assumptions, the $\gamma$-ray flux predictions still have a large uncertainty, mainly due to the dependence of the flux from the emission volume (i.e. from $R$ and $\Gamma$ ). In our model, however, these are not completely free parameters, since on one hand they must be compatible with the variability timescales, and on the other they control the injection timescale $t_{\mathrm{inj}}$. The dependence of the inverse Compton flux from $R$ and $\Gamma$ is therefore complex. For instance, for a given $L^{\prime}$ and $B$, an increase of $R$ makes the Compton flux to decrease $\left(\propto R^{-2}\right)$, but it makes also $t_{\text {inj }}$ to increase, leading to a smaller value of $\gamma_{\mathrm{c}}\left(=\gamma_{\text {peak }}\right)$, and then to a smaller synchrotron peak frequency. To compensate for that, it would be necessary to decrease $B$ (to increase the cooling time), but this leads to an increase of the Compton flux.

Our choice has been to use values of $R$ around $\sim 10^{16} \mathrm{~cm}$, which are the typical values obtained for sources with good monitoring and with SEDs well sampled also at high energies, like Mkn 421 and 1ES 2155-304. 
Table 2. TeV BL Lac candidates. S: Slew survey sample; Ex: Exosat sample; R: RGB sample; Ra: RASS sample; 1J: 1 Jy sample; oth.: others catalogues, see Donato et al. (2001) and references therein. None of the above sources is in the EMSS sample. The references report the basic source of data for the SEDs, in addition to those in the NED database and the respective sample papers (Sect. 4.1). All the MeV-GeV upper limits are from Fichtel et al. (1994) (first EGRET catalogue). Ah00: Aharonian et al. (2000); Ba94: Bade et al. (1994); Ba00: Bauer et al. (2000); Be92: Bersanelli et al. (1992); Be01: Beckman et al. (2001); Bk99: Buckley (1999); Br89: Brown et al. (1989); Br95: Brinkmann et al. (1995); Br97: Brinkmann et al. (1997); BS94: Brinkmann \& Siebert (1994); C97: Comastri et al. (1997); Ca97: Catanese et al. (1997a); Ch99: Chadwick et al. (1999); Ci95: Ciliegi et al. (1995); Co01: Costamante et al. (2001); Di96: Dingus et al. (1996); E94: Edelson et al. (1994); F96: Fruscione (1996); Fa90: Falomo et al. (1990); Fa94: Falomo et al. (1994); Fo98: Fossati et al. (1998); GAM95: Giommi et al. (1995); Ge94: Gear et al. (1994); GS95: Ghosh \& Soundararajaperumal (1995); GT96: George \& Turner (1996); IN88: Inpey \& Neugebauer (1988); Ke95: Kerrick et al. (1995); Ki97: Kifune et al. (1997); La96: Lamer et al. (1996); Li94: Litchfield et al. (1994); Ma88: Madejski et al. (1988); Ma99: Madejski et al. (1999); Ne94: Neumann et al. (1994); Pi93: Pian et al. (1993); Pir00: F. Piron Ph.D. Thesis, 2000; P01: Padovani et al. (2001); Ro99: Roberts et al. (1999); Ro01: Robson et al. (2001); Sa94: Sambruna et al. (1994); SG99: Stevens \& Gear (1999); St94: Stevens et al. (1994); Si91: Sitko \& Sitko (1991); Ta01: Tagliaferri et al. (2001) and references therein; Te98: Terasranta et al. (1998); To96: Tornikoski et al. (1996); Wo00: Wolter et al. (2000) and references therein; WW90: Worrall \& Wilkes (1990).

\begin{tabular}{|c|c|c|c|c|c|c|c|c|c|c|c|}
\hline Name & $\mathrm{S}$ & Ex & $\overline{\mathrm{Ra}}$ & $\mathrm{R}$ & $1 \mathrm{~J}$ & oth. & $z$ & $\begin{array}{l}F_{5 \mathrm{GHz}} \\
\mathrm{Jy}\end{array}$ & $\begin{array}{l}F_{5500} \AA \\
\text { mJy }\end{array}$ & $\begin{array}{l}F_{1 \mathrm{keV}} \\
\mu \mathrm{Jy} \\
\end{array}$ & Ref \\
\hline $0033+595$ & $\mathrm{X}$ & & & $\mathrm{X}$ & & & $0.086^{a}$ & 0.066 & 2.95 & 5.66 & Co01, SG99, Br97 \\
\hline $0120+340$ & $\mathrm{X}$ & & & $\mathrm{X}$ & & & 0.272 & 0.045 & 1.23 & 4.73 & Co01, BS94 \\
\hline $0136+391$ & & & $\mathrm{X}$ & $\mathrm{X}$ & & & - & 0.049 & 3.41 & 2.39 & $\mathrm{Ba00,} \mathrm{Br} 97$ \\
\hline $0214+517$ & & & $\mathrm{X}$ & $\mathrm{X}$ & & & 0.049 & 0.161 & 0.39 & 1.60 & Ne94 \\
\hline $0219+428$ & $\mathrm{X}$ & $\mathrm{X}$ & & & & & 0.444 & 0.806 & 5.12 & 1.57 & Pi93, Si91, Ro00, WW90, C97, Di96 \\
\hline $0229+200$ & $\mathrm{X}$ & & & & & & 0.139 & 0.049 & 9.93 & 2.88 & Ah00 \\
\hline $0323+022$ & $\mathrm{X}$ & $\mathrm{X}$ & & $\mathrm{X}$ & & & 0.147 & 0.042 & 0.81 & 4.49 & Fo98, SG99, Be01, Ch99 \\
\hline $0414+009$ & $\mathrm{X}$ & $\mathrm{X}$ & & $\mathrm{X}$ & & & 0.287 & 0.070 & 0.82 & 9.29 & Wo00, Ah00 \\
\hline $0548-322$ & $\mathrm{X}$ & $\mathrm{X}$ & & & & & 0.069 & 0.170 & 3.61 & 7.47 & Co01, GAM95, WW90, Ta95, Ti94, Ro99, Bk99 \\
\hline $0556-384$ & & & & & & & 0.034 & 0.068 & 0.56 & 2.2 & SG96, GT96, Ci95 \\
\hline $0647+250$ & $\mathrm{X}$ & & & $\mathrm{X}$ & & & - & 0.073 & 5.12 & 6.01 & SG99, GAM95, BS94, Ah00 \\
\hline $0806+524$ & $\mathrm{X}$ & & & $\mathrm{X}$ & & & 0.138 & 0.172 & 6.33 & 3.51 & SG99, GAM95, BS94 \\
\hline $0809+024$ & & & & & & $\mathrm{X}$ & - & 0.071 & 6.23 & 1.85 & $\mathrm{Ba} 00, \mathrm{Br} 97$ \\
\hline $0851+203$ & $\mathrm{X}$ & $\mathrm{X}$ & & $\mathrm{X}$ & $\mathrm{X}$ & & 0.306 & 1.740 & 14.9 & 1.70 & $\begin{array}{l}\text { To96, Br89, IN88, Ge94, } \\
\text { E94, Li94, Sa94, Ma88, P01 }\end{array}$ \\
\hline $1011+496$ & $\mathrm{X}$ & & $\mathrm{X}$ & $\mathrm{X}$ & & & 0.200 & 0.286 & 2.04 & 1.38 & Fo98 \\
\hline $1028+511$ & $\mathrm{X}$ & & $\mathrm{X}$ & $\mathrm{X}$ & & & 0.361 & 0.044 & 1.29 & 4.80 & Fo98, Be01 \\
\hline $1101-232$ & $\mathrm{X}$ & & & & & & 0.186 & 0.066 & 2.52 & 9.25 & Wo00, SG99, Ch99 \\
\hline $1114+202$ & & & & & & $\mathrm{X}$ & 0.139 & 0.074 & 3.03 & 7.31 & Co01, Br97 \\
\hline $1133+704$ & $\mathrm{X}$ & $\mathrm{X}$ & $\mathrm{X}$ & $\mathrm{X}$ & & & 0.045 & 0.274 & 9.75 & 4.56 & IN88, La96, Pir00, Bk99 \\
\hline $1136.5+6737$ & & & $\mathrm{X}$ & $\mathrm{X}$ & & & 0.135 & 0.040 & 0.49 & 3.40 & F96, Ba94 \\
\hline $1215+303$ & $\mathrm{X}$ & $\mathrm{X}$ & $\mathrm{X}$ & $\mathrm{X}$ & & & 0.237 & 0.445 & 3.08 & 3.7 & GAM95, Fo98, Te98 \\
\hline $1218+304$ & $\mathrm{X}$ & $\mathrm{X}$ & $\mathrm{X}$ & $\mathrm{X}$ & & & 0.182 & 0.056 & 1.63 & 6.39 & Co01, GAM95, Pi93, Sa94, Fo98, Pir00 \\
\hline $1417+257$ & & & $\mathrm{X}$ & $\mathrm{X}$ & & & 0.237 & 0.040 & 0.56 & 2.65 & $\mathrm{Ba00,} \mathrm{La96}$ \\
\hline $1440+122$ & $\mathrm{X}$ & & & $\mathrm{X}$ & & & 0.162 & 0.041 & 0.90 & 1.47 & $\mathrm{Ba} 00, \mathrm{Br} 95$ \\
\hline $1544+820$ & $\mathrm{X}$ & & & & & & - & 0.043 & 1.15 & 2.31 & GAM95, Fo98, Be01 \\
\hline $1553+113$ & $\mathrm{X}$ & & & $\mathrm{X}$ & & & 0.360 & 0.636 & 6.15 & 6.54 & GAM95, Ne94, Fa90, IN88, Be01 \\
\hline $1722+119$ & & $\mathrm{X}$ & $\mathrm{X}$ & $\mathrm{X}$ & & & 0.018 & 0.088 & 2.95 & 4.02 & Be92, Pi93, GAM95, SG99, Sa94 \\
\hline $1727+502$ & $\mathrm{X}$ & $\mathrm{X}$ & & $\mathrm{X}$ & & & 0.055 & 0.159 & 1.27 & 2.73 & Fo98, Pi93, Ke95, Bk99 \\
\hline $1741+196$ & $\mathrm{X}$ & & & $\mathrm{X}$ & & & 0.084 & 0.223 & 1.86 & 2.88 & Br97, Pir00 \\
\hline $1959+650$ & $\mathrm{X}$ & & & & & & 0.047 & 0.252 & 1.35 & 9.29 & GAM95, SG99, F96, Be01, Bk99 \\
\hline $2005-489$ & $\mathrm{X}$ & $\mathrm{X}$ & & & $\mathrm{X}$ & & 0.071 & 1.192 & 4.84 & 5.42 & Ta01, Ki99, Bk99 \\
\hline $2200+420$ & $\mathrm{X}$ & $\mathrm{X}$ & & $\mathrm{X}$ & $\mathrm{X}$ & & 0.069 & 3.593 & 17.27 & 1.91 & $\begin{array}{l}\text { St94, Br89, IN88, To96, Li94, Bk99, } \\
\text { Ca97, Sa99, Ma99, P01 }\end{array}$ \\
\hline $2356-309$ & & & & & & $\mathrm{X}$ & 0.165 & 0.065 & 0.67 & 5.78 & Co01, Be92, Fa94 \\
\hline
\end{tabular}

${ }^{a}$ Tentative redshift, Perlman priv. comm. (see NED notes).

Another point of uncertainty is the exact determination of $\nu_{\text {peak }}$ and the corresponding synchrotron peak flux. For many sources this is provided by the BeppoSAX observations, which can be fitted by a broken power law, immediately yielding $\nu_{\text {peak }}$. For other sources of poorly known
SED the determination of $\nu_{\text {peak }}$ is more uncertain, and for these sources the predicted SEDs of course suffer from this uncertainty (see e.g. the SED of $0214+517,1440+122$ and $1544+820$, for which there is no information of the slope 
Table 3. Input parameters for the SSC model, resulting values of $\gamma_{\text {peak }}$, and predicted fluxes at high energies, above $40 \mathrm{GeV}$, $0.3 \mathrm{TeV}$ and $1 \mathrm{TeV}$, according to the parameterization of the SED adapted from Fossati et al. (1998) (first number) and according to the SSC model discussed in Sect. 5.1 (second number). $L^{\prime}$ is the intrinsic power (i.e. measured in the comoving frame), $R$ the cross sectional radius of the emitting region, $\Gamma$ is the bulk Lorentz factor, $\theta$ is the viewing angle, $n$ the slope of the particle distribution above the cooling energy $\gamma_{\mathrm{c}}$ (see text), $\gamma_{1}$ and $\gamma_{2}$ are the extreme Lorentz factors of the injected particle distribution and $\gamma_{\text {peak }}$ is the particle Lorentz factor of the electrons emitting most of the radiation (i.e. at the synchrotron and self-Compton peaks). ${ }^{a} z=0.2$ assumed. ${ }^{b}$ Fluxes in units of $10^{-11}$ photons $\mathrm{cm}^{-2} \mathrm{~s}^{-1}$.

\begin{tabular}{|c|c|c|c|c|c|c|c|c|c|c|c|c|}
\hline Name & $\begin{array}{l}L^{\prime} \\
\operatorname{erg~s}^{-1}\end{array}$ & $\begin{array}{l}R \\
\mathrm{~cm}\end{array}$ & $\begin{array}{l}B \\
\mathrm{G}\end{array}$ & $\Gamma$ & $\theta$ & $n$ & $\gamma_{1}$ & $\gamma_{\text {peak }}$ & $\gamma_{2}$ & $F_{(>40 \mathrm{GeV})}^{b}$ & $F_{(>0.3 \mathrm{TeV})}^{b}$ & $F_{(>1 \mathrm{TeV})}^{b}$ \\
\hline $0033+595$ & $1.0 \mathrm{e} 41$ & $9.0 \mathrm{e} 15$ & 0.8 & 14 & 3.0 & 3.01 & 1000 & $5.3 \mathrm{e} 4$ & $6.0 \mathrm{e} 5$ & $15.0 / 2.93$ & $2.04 / 0.25$ & $0.48 / 0.04$ \\
\hline $0120+340$ & $4.4 \mathrm{e} 41$ & $1.0 \mathrm{e} 16$ & 0.6 & 15 & 3.0 & 3.2 & 700 & $7.0 \mathrm{e} 4$ & $2.5 \mathrm{e} 5$ & $3.17 / 4.90$ & $0.28 / 0.30$ & $0.06 /-$ \\
\hline $0136+391^{a}$ & $3.0 \mathrm{e} 42$ & $1.0 \mathrm{e} 16$ & 2.0 & 15 & 4.8 & 3.6 & 500 & $4.5 \mathrm{e} 3$ & $5.0 \mathrm{e} 5$ & $5.22 / 4.00$ & $0.56 / 0.14$ & $0.12 / 2.7 \mathrm{e}-3$ \\
\hline $0214+517$ & $5.0 \mathrm{e} 40$ & $8.0 \mathrm{e} 15$ & 0.4 & 13 & 5.0 & 3.95 & 1500 & $1.7 \mathrm{e} 5$ & $6.0 \mathrm{e} 5$ & $43.4 / 1.59$ & $5.93 / 0.07$ & $1.43 / 6.2 \mathrm{e}-3$ \\
\hline $0219+428$ & $5.0 \mathrm{e} 42$ & $2.0 \mathrm{e} 16$ & 1.4 & 15 & 3.0 & 3.4 & 700 & $4.7 \mathrm{e} 3$ & $8.0 \mathrm{e} 4$ & $7.01 / 9.62$ & $0.14 /-$ & $0.01 /-$ \\
\hline $0229+200$ & $1.5 \mathrm{e} 41$ & $1.0 \mathrm{e} 16$ & 0.3 & 15 & 3.5 & 3.5 & 700 & $2.8 \mathrm{e} 5$ & $4.0 \mathrm{e} 5$ & $7.67 / 3.81$ & $0.96 / 0.31$ & $0.21 / 4.0 \mathrm{e}-3$ \\
\hline $0323+022$ & $1.0 \mathrm{e} 41$ & $1.0 \mathrm{e} 16$ & 0.9 & 11 & 3.7 & 3.5 & 800 & $2.7 \mathrm{e} 4$ & $1.8 \mathrm{e} 5$ & $6.65 / 1.18$ & $0.84 / 0.01$ & $0.18 /-$ \\
\hline $0414+009$ & $8.0 \mathrm{e} 41$ & $1.0 \mathrm{e} 16$ & 1.5 & 14 & 3.5 & 3.2 & 500 & $9.8 \mathrm{e} 3$ & $1.6 \mathrm{e} 5$ & $2.91 / 3.42$ & $0.23 / 0.07$ & $0.04 /-$ \\
\hline $0548-322$ & $7.5 \mathrm{e} 40$ & $8.0 \mathrm{e} 15$ & 0.8 & 12 & 4.0 & 3.1 & 700 & $5.0 \mathrm{e} 4$ & $5.0 \mathrm{e} 5$ & $31.9 / 1.56$ & $4.14 / 0.10$ & $0.91 / 0.015$ \\
\hline $0556-383$ & $7.0 \mathrm{e} 40$ & $8.0 \mathrm{e} 15$ & 0.4 & 16 & 5.0 & 3.2 & 1500 & $2.5 \mathrm{e} 5$ & $2.5 \mathrm{e} 5$ & $37.8 / 5.51$ & $5.84 / 0.42$ & $1.56 /-$ \\
\hline $0647+250^{a}$ & $1.0 \mathrm{e} 42$ & $1.2 \mathrm{e} 16$ & 1.4 & 16 & 3.3 & 3.6 & 500 & $1.0 \mathrm{e} 4$ & $2.0 \mathrm{e} 5$ & $6.16 / 8.74$ & $0.59 / 0.24$ & $0.12 /-$ \\
\hline $0806+524$ & $1.0 \mathrm{e} 42$ & $1.2 \mathrm{e} 16$ & 1.5 & 15 & 4.0 & 3.4 & 300 & $8.5 \mathrm{e} 3$ & $7.0 \mathrm{e} 4$ & $14.7 / 10.7$ & $1.36 /-$ & $0.27 /-$ \\
\hline $0809+024^{a}$ & $8.0 \mathrm{e} 41$ & $1.0 \mathrm{e} 16$ & 1.3 & 14 & 4.8 & 3.3 & 1000 & $1.2 \mathrm{e} 4$ & $2.0 \mathrm{e} 5$ & $6.08 / 2.20$ & $0.58 / 0.04$ & $0.12 /-$ \\
\hline $0851+202$ & $5.0 \mathrm{e} 42$ & $1.3 \mathrm{e} 16$ & 5.0 & 13 & 3.5 & 3.4 & 150 & $6.5 \mathrm{e} 2$ & 7.e3 & $23.7 /-$ & $0.42 /-$ & $0.03 /-$ \\
\hline $1028+511$ & $2.7 \mathrm{e} 41$ & $1.0 \mathrm{e} 16$ & 1.0 & 14 & 3.0 & 3.2 & 800 & $2.6 \mathrm{e} 4$ & $2.0 \mathrm{e} 5$ & $7.15 / 3.89$ & $0.43 /-$ & $0.06 /-$ \\
\hline $1011+496$ & $7.0 \mathrm{e} 41$ & $2.0 \mathrm{e} 16$ & 0.7 & 12 & 4.0 & 3.4 & 300 & $1.9 \mathrm{e} 4$ & $1.0 \mathrm{e} 5$ & $1.67 / 3.31$ & $0.12 / 0.14$ & $0.02 /-$ \\
\hline $1101-232$ & $5.3 \mathrm{e} 41$ & $7.0 \mathrm{e} 15$ & 0.9 & 16 & 2.8 & 3.1 & 300 & $5.3 \mathrm{e} 4$ & $1.0 \mathrm{e} 6$ & $6.67 / 10.2$ & $0.67 / 0.93$ & $0.15 / 0.18$ \\
\hline $1114+202$ & $4.5 \mathrm{e} 41$ & $8.0 \mathrm{e} 15$ & 1.5 & 16 & 3.0 & 4.6 & 6000 & $6.0 \mathrm{e} 3$ & $2.5 \mathrm{e} 5$ & $10.1 / 8.51$ & $1.17 / 0.10$ & $0.28 /-$ \\
\hline $1133+704$ & $2.0 \mathrm{e} 41$ & $2.0 \mathrm{e} 16$ & 0.8 & 10 & 5.0 & 3.7 & 400 & $1.6 \mathrm{e} 4$ & $1.7 \mathrm{e} 5$ & $62.8 / 2.15$ & $8.50 / 0.03$ & $1.93 /-$ \\
\hline $1136+673$ & $4.0 \mathrm{e} 41$ & $1.0 \mathrm{e} 16$ & 1.0 & 15 & 4.2 & 3.5 & 1000 & $2.4 \mathrm{e} 4$ & $1.5 \mathrm{e} 5$ & $7.30 / 5.40$ & $0.92 / 0.10$ & $0.21 /-$ \\
\hline $1215+303$ & $1.0 \mathrm{e} 41$ & $1.0 \mathrm{e} 16$ & 2.5 & 11 & 4.6 & 3.8 & 300 & $3.4 \mathrm{e} 3$ & $4.0 \mathrm{e} 4$ & $4.06 / 0.07$ & $0.16 /-$ & $0.02 /-$ \\
\hline $1218+304$ & $2.0 \mathrm{e} 41$ & $1.0 \mathrm{e} 16$ & 1.5 & 16 & 2.9 & 3.9 & 600 & $1.4 \mathrm{e} 4$ & $3.0 \mathrm{e} 5$ & $6.36 / 5.82$ & $0.67 / 0.16$ & $0.15 /-$ \\
\hline $1417+257$ & $1.7 \mathrm{e} 42$ & $1.0 \mathrm{e} 16$ & 1.5 & 15 & 4.2 & 3.4 & 400 & $8.4 \mathrm{e} 3$ & $2.5 \mathrm{e} 5$ & $3.76 / 6.88$ & $0.38 / 0.21$ & $0.08 /-$ \\
\hline $1440+122$ & $2.6 \mathrm{e} 41$ & $1.2 \mathrm{e} 16$ & 0.7 & 15 & 4.2 & 3.5 & 500 & $4.6 \mathrm{e} 4$ & $5.0 \mathrm{e} 5$ & $6.11 / 1.89$ & $0.78 / 0.09$ & $0.20 / 0.01$ \\
\hline $1544+820^{a}$ & $6.0 \mathrm{e} 41$ & $8.0 \mathrm{e} 15$ & 1.2 & 14 & 4.0 & 3.3 & 400 & $1.8 \mathrm{e} 4$ & $4.0 \mathrm{e} 5$ & $4.89 / 5.37$ & $0.54 / 0.22$ & $0.12 /-$ \\
\hline $1553+113$ & $2.5 \mathrm{e} 42$ & $3.0 \mathrm{e} 16$ & 0.7 & 15 & 2.5 & 3.6 & 300 & $1.3 \mathrm{e} 4$ & $1.0 \mathrm{e} 5$ & $8.92 / 22.3$ & $0.20 / 0.42$ & $0.02 /-$ \\
\hline $1722+119$ & $4.0 \mathrm{e} 40$ & $1.5 \mathrm{e} 16$ & 0.7 & 10 & 6.5 & 3.6 & 600 & $2.6 \mathrm{e} 4$ & $5.0 \mathrm{e} 5$ & $76.6 / 1.06$ & $12.8 / 0.015$ & $3.52 / 1.0 \mathrm{e}-3$ \\
\hline $1727+502$ & $1.1 \mathrm{e} 41$ & $1.0 \mathrm{e} 16$ & 0.8 & 10 & 5.0 & 3.5 & 600 & $3.0 \mathrm{e} 4$ & $1.9 \mathrm{e} 5$ & $38.7 / 2.64$ & $5.19 / 0.07$ & $1.23 /-$ \\
\hline $1741+196$ & $1.0 \mathrm{e} 41$ & $1.0 \mathrm{e} 16$ & 0.4 & 13 & 4.0 & 3.8 & 400 & $1.3 \mathrm{e} 5$ & $6.0 \mathrm{e} 5$ & $31.6 / 4.31$ & $3.59 / 0.29$ & $0.84 / 0.01$ \\
\hline $1959+650$ & $8.0 \mathrm{e} 40$ & $1.0 \mathrm{e} 16$ & 1.2 & 13 & 4.0 & 3.6 & 500 & $1.9 \mathrm{e} 4$ & $1.5 \mathrm{e} 5$ & $56.7 / 2.08$ & $7.46 / 0.03$ & $1.74 /-$ \\
\hline $2005-489$ & $3.5 \mathrm{e} 41$ & $1.0 \mathrm{e} 16$ & 1.2 & 16 & 3.0 & 3.3 & 600 & $2.1 \mathrm{e} 4$ & $5.0 \mathrm{e} 5$ & $67.1 / 62.5$ & $5.14 / 2.67$ & $0.90 / 0.17$ \\
\hline $2200+428$ & $1.3 \mathrm{e} 42$ & $5.0 \mathrm{e} 15$ & 1.2 & 14 & 3.3 & 4.3 & 1000 & $1.0 \mathrm{e} 3$ & $2.0 \mathrm{e} 5$ & $67.7 / 42.8$ & $3.32 / 0.17$ & $0.43 /-$ \\
\hline $2356-309$ & $2.0 \mathrm{e} 41$ & $7.0 \mathrm{e} 15$ & 1.2 & 16 & 3.0 & 3.01 & 300 & $6.0 \mathrm{e} 4$ & $7.0 \mathrm{e} 5$ & $7.64 / 3.30$ & $0.84 / 0.19$ & $0.12 / 0.03$ \\
\hline
\end{tabular}

of the X-ray spectra and only a few observations in the IR-optical bands).

The applied model is aimed to reproduce the spectrum originating in a limited part of the jet, thought to be responsible of most of the emission. This region is necessarily compact, since it must account for the fast variability shown by all blazars, especially at high frequencies. Therefore the radio emission from this compact regions is strongly self-absorbed, and the model cannot account for the observed radio flux.

\subsection{Fossati et al. (1998) description of the SED}

Fossati et al. (1998) proposed a simple phenomenological description of the average SED of blazars based on their bolometric observed luminosity, thought to be well traced by the radio luminosity. In their parameterization, the radio luminosity is assumed to be the key parameter, determining the peak frequency of the synchrotron spectrum and the relative importance of the inverse Compton power. More recently, Donato et al. (2001) have revisited this parameterization, assuming a slightly different relation between the radio power, the synchrotron peak frequency and the inverse Compton luminosity, but only for objects below a radio luminosity of $10^{43} \mathrm{erg} \mathrm{s}^{-1}$. With this new parameterization, objects of low power are assumed to have equal luminosities in the synchrotron and in the self-Compton components of their spectra, and the ratio of the peak frequencies $\left(\nu_{\mathrm{c}} / \nu_{\mathrm{s}}\right)$ was allowed to increase for increasing $\nu_{\mathrm{s}}$ (as expected in a SSC scenario).

To improve this parameterization also for objects with even lower radio luminosity (i.e. higher $\nu_{\text {peak }}$ ), we have 


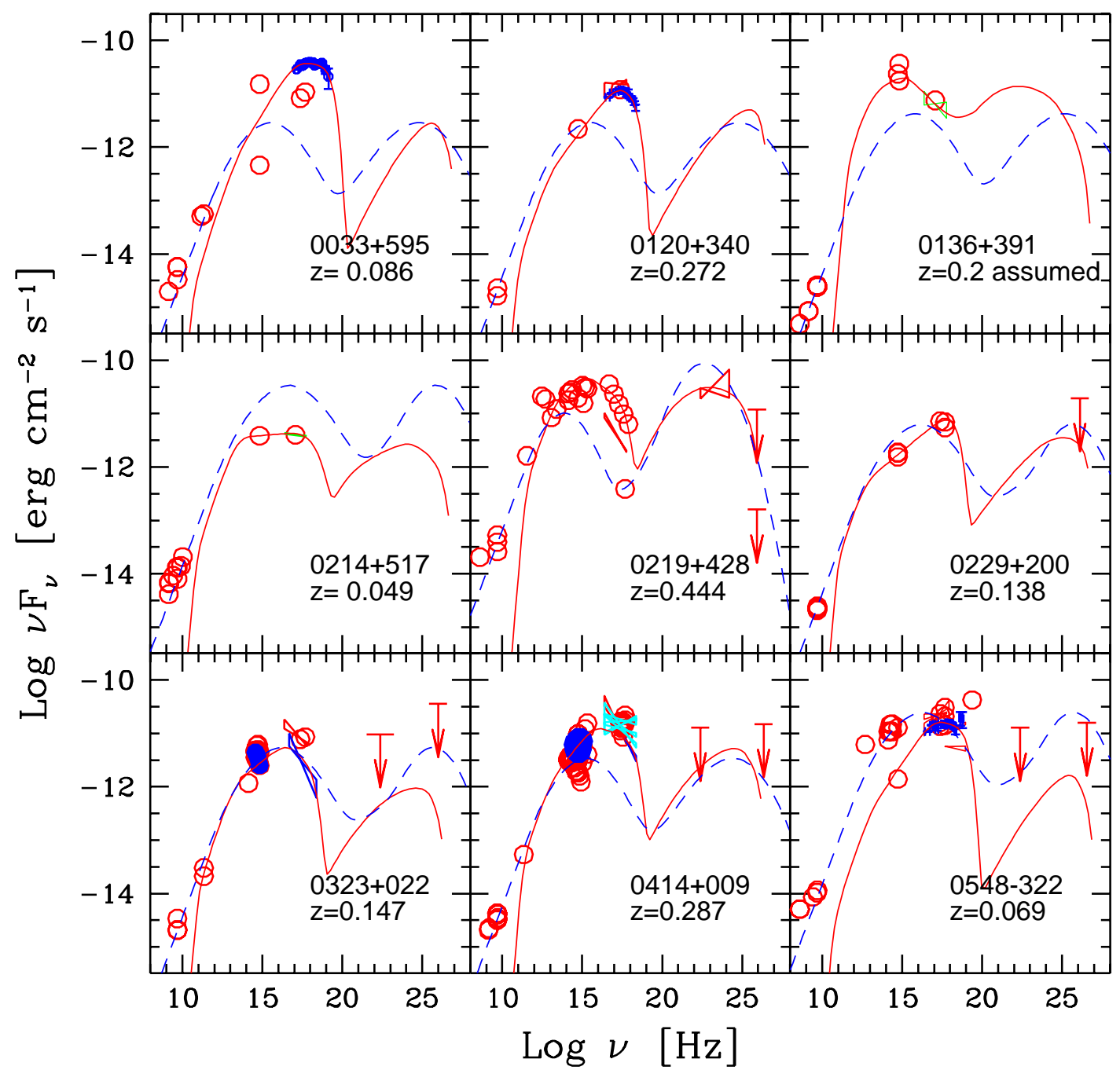

Fig. 6. a) SEDs of our best candidates for TeV emission. The solid lines refer to the SSC model as explained in Sect. 5.1. Dashed lines correspond to the phenomenological prescription of Fossati et al. (1998), as slightly modified by Donato et al. (2001) and in this paper. Sources of data listed in Table 2.

further modified the Donato et al. prescription for sources with radio luminosity below $10^{41.2} \mathrm{erg} \mathrm{s}^{-1}$, to mimic some effects of the Klein-Nishina regime. The first modification involves the ratio $\nu_{\mathrm{c}} / \nu_{\mathrm{s}}$, since in the Klein-Nishina regime, $\nu_{\mathrm{s}} \propto \gamma_{\text {peak }}^{2}$ while $\nu_{\mathrm{c}} \propto \gamma_{\text {peak. }}$. Then the ratio $\nu_{\mathrm{c}} / \nu_{\mathrm{s}} \propto \gamma_{\text {peak }}^{-1} \propto \nu_{\mathrm{s}}^{-1 / 2}$. Below $L_{\mathrm{R}}=10^{41.2} \mathrm{erg} \mathrm{s}^{-1}$, we then decrease $\nu_{\mathrm{c}} / \nu_{\mathrm{s}}$ assuming that $\log \left(\nu_{\mathrm{c}} / \nu_{\mathrm{s}}\right)=9.4-$ $0.8 \times\left[41.2-\log L_{\mathrm{R}}\right]$. The other modification concerns the width of the parabola representing the Compton peak, which is reduced with respect to the synchrotron one $\left(\sigma_{\text {Comp }}^{2}=\sigma_{\text {sync }}^{2} / 2\right.$; see Fossati et al. 1997, Appendix A, and F98 for a description of the parameters). In this way the resulting SED shapes provide a better representation of the individual SEDs for the highest $\nu_{\text {peak }}$ sources.

We have applied this parameterization to all the objects in our sample, and the resulting fits are shown as dashed lines in Fig. 6. From these we obtained the photon fluxes listed in Table 3, integrating above the corresponding threshold energy.
From the comparison with the homogeneous SSC models, we can see that the F98 parameterization tends to overestimate the Compton $\gamma$-ray emission, although it agrees with the existing upper limits in the $\mathrm{GeV}$ and $\mathrm{TeV}$ bands in all cases but $0851+202,1133+704$ and $1553+113$. This is not unexpected, since, by construction, the Compton power is never less than the synchrotron one and the assumed parabolic shape does not account for sharp high energy cut-offs. This parameterization however, which is built to describe the average SED of sources of equal synchrotron and self-Compton power, is in good agreement with the flux measurements of the already detected TeV sources (see Fig. 1).

\section{Discussion and conclusions}

With new Cherenkov telescopes foreseen to operate in the next few years the TeV extragalactic astronomy is entering its adulthood. A tenfold increase in sensitivity (expected 


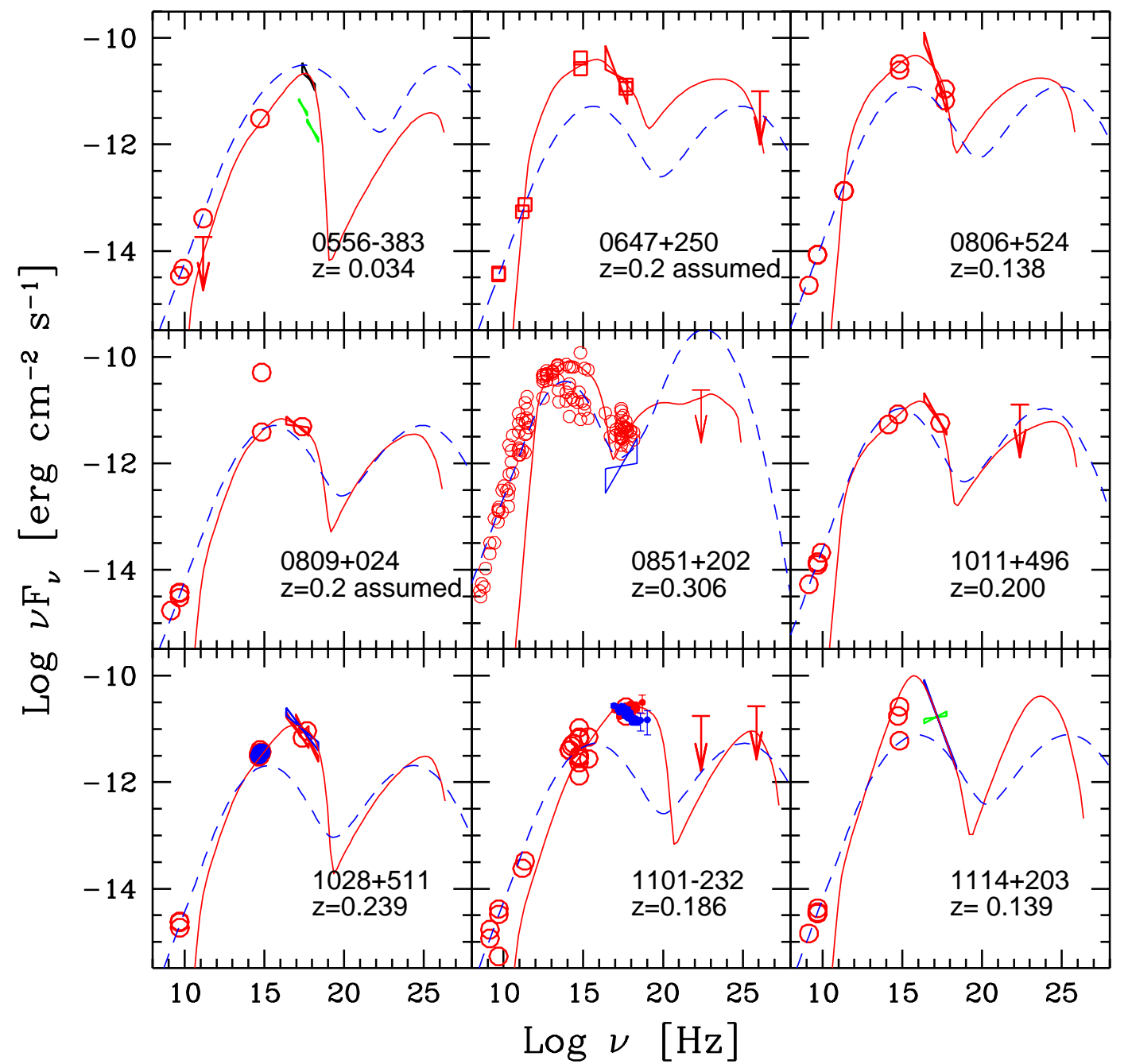

Fig. 6. b) SEDs of our best candidates for TeV emission. The solid lines refer to the SSC model as explained in Sect. 5.1. Dashed lines correspond to the phenomenological prescription of Fossati et al. (1998), as slightly modified by Donato et al. (2001) and in this paper. Sources of data listed in Table 2.

for the forthcoming installations) would mean the possible detection of $\sim 100$ BL Lacs, if the counts of BL Lac object at $\mathrm{TeV}$ energies are roughly Euclidean (at the bright flux end) and neglecting absorption by IRB. Because of IRB absorption, the counts will be flatter than Euclidean, but the lower energy threshold of some new instruments may compensate for the extragalactic absorption, as well as favoring the detection of slightly less blue objects. Therefore many more sources are expected to be detectable by the new telescopes, and this motivated us to study which kind of BL Lac objects is more likely to be detected at high energies.

Our findings can be summarized very simply once we realize that, to produce a strong $\mathrm{TeV}$ emission, the inverse Compton process needs a sufficient number of both very high energy electrons and soft seed photons. Therefore we require both a strong $\mathrm{X}$-ray flux and a sufficiently strong radio-through-optical flux. Since the optical flux can be contaminated, especially in low redshift sources, by the underlying host galaxy, our sources are primarily selected as bright both in the $\mathrm{X}$-ray and radio bands. All but one of these sources are also bright in the optical.

With respect to the previous work by Stecker et al. (1996), our criterium introduces the further requirement that the source must be a relatively strong radio emitter. The other difference is that we considered not only the Einstein Slew survey sample of BL Lacs, but several other BL Lac samples.

Besides selecting the best candidates through their location in the radio - X-ray flux plane (a criterium largely model independent), we have also tried to quantify the level of the expected high energy emission for each selected source, by applying a one-zone synchrotron self-Compton model and also the phenomenological description of the SED of Fossati et al. (1998), slightly modified to better account of the average SED of low power BL Lacs. The latter model, by construction, assumes equal power between the synchrotron and the inverse Compton components of the SEDs, and almost always predicts larger high energy fluxes than the SSC model. In the SSC model, in 


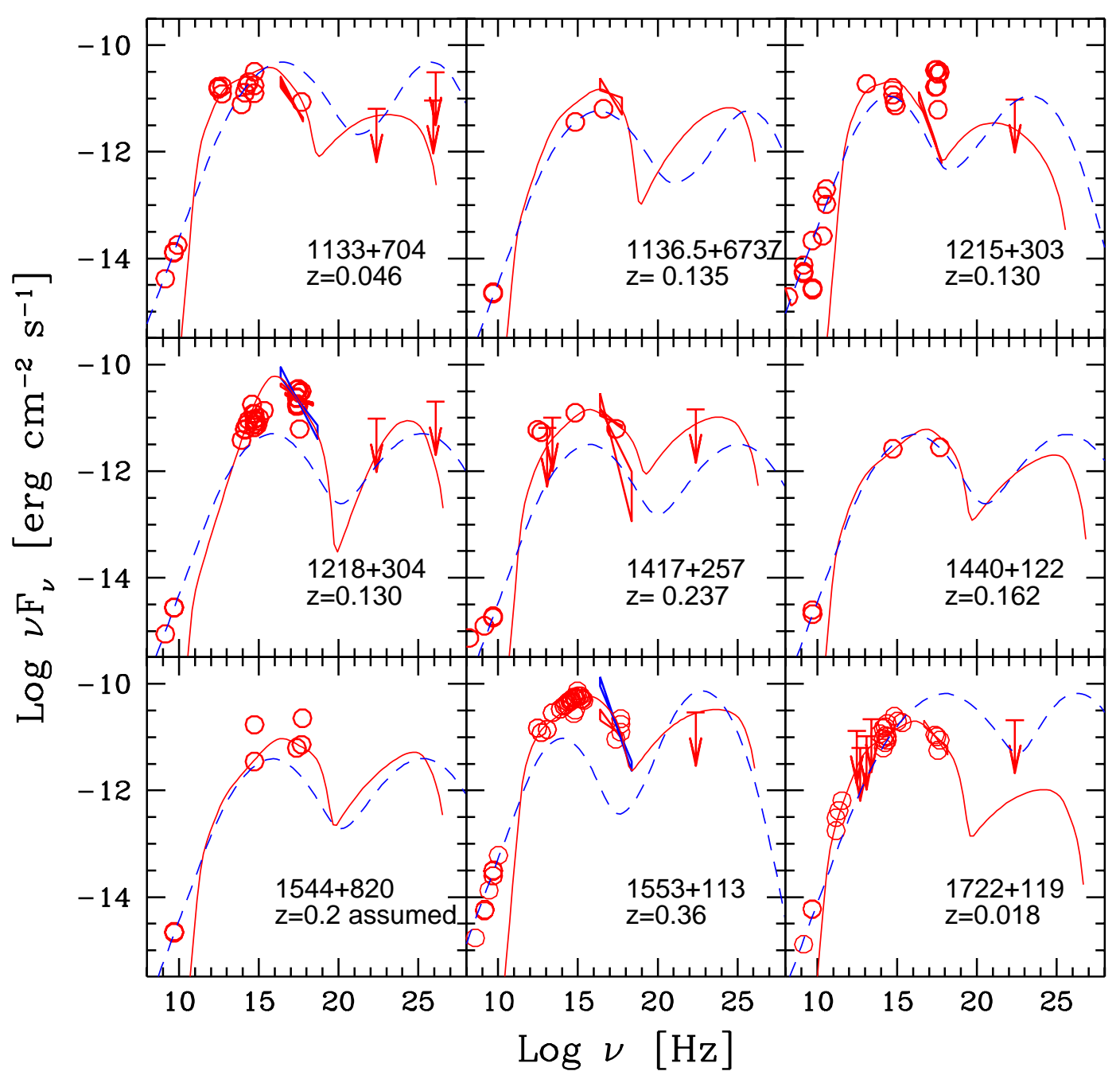

Fig. 6. c) SEDs of our best candidates for TeV emission. The solid lines refer to the SSC model as explained in Sect. 5.1. Dashed lines correspond to the phenomenological prescription of Fossati et al. (1998), as slightly modified by Donato et al. (2001) and in this paper. Sources of data listed in Table 2.

fact, the Compton dominance is not fixed a priori, but found by fitting the synchrotron part of the spectrum, which fixes the value of the magnetic field. We stress that the F98 prescription was designed including also the SEDs of those BL Lacs already detected in the TeV band (and indeed is in good agreement with their $\mathrm{TeV}$ flux levels), and therefore seems more appropriate to predict the $\mathrm{TeV}$ flux of sources in high state. The adopted SSC model, instead, is designed to fit the known synchrotron part of the SED, which is often representative of a more "normal" or quiescent state. This explains the sometimes large discrepancy between the predicted fluxes of the two models. Since BL Lac objects are among the most variable sources, especially at high energies, the two foreseen flux levels could be thought of as an approximate range of variability, and the average flux could be considered as a measure of the probability to find the source in a particular $\mathrm{TeV}$ state.

We would like to stress, anyway, that the uncertainties on the key parameters we used for the model and the nonsimultaneity of the fitted data lead to large uncertainties in the predicted $\mathrm{TeV}$ flux, sometimes of the same order of the differences between the two adopted models. Our predicted fluxes, therefore, also in the case of the SSC model, must be considered as "best guesses" on the high energy emission from these objects. Within these limits, the SSC model provides more information than the phenomenological parameterization, since it gives also the expected shape of the high energy spectrum.

Since the level of the synchrotron X-ray flux measures, in our scenario (as well as in the Stecker et al. 1996 one), the number of $\mathrm{TeV}$ energy electrons, the $\mathrm{X}$-ray monitoring of our candidates is particularly useful to catch sources in high $\mathrm{TeV}$ states, as already partially done through the All Sky Monitor (ASM) onboard the RossiXTE satellite.

Besides the fluxes above $300 \mathrm{GeV}$, the most common threshold of present Cherenkov telescopes, we also give our estimates above $40 \mathrm{GeV}$, which is approximately the energy threshold of CELESTE and of forthcoming observatories like HESS and MAGIC (with VERITAS at $\sim 50 \mathrm{GeV}$, Weekes 1999). Emission at these energies is much less absorbed by the cosmic infrared background, giving the opportunity to see more distant sources and 


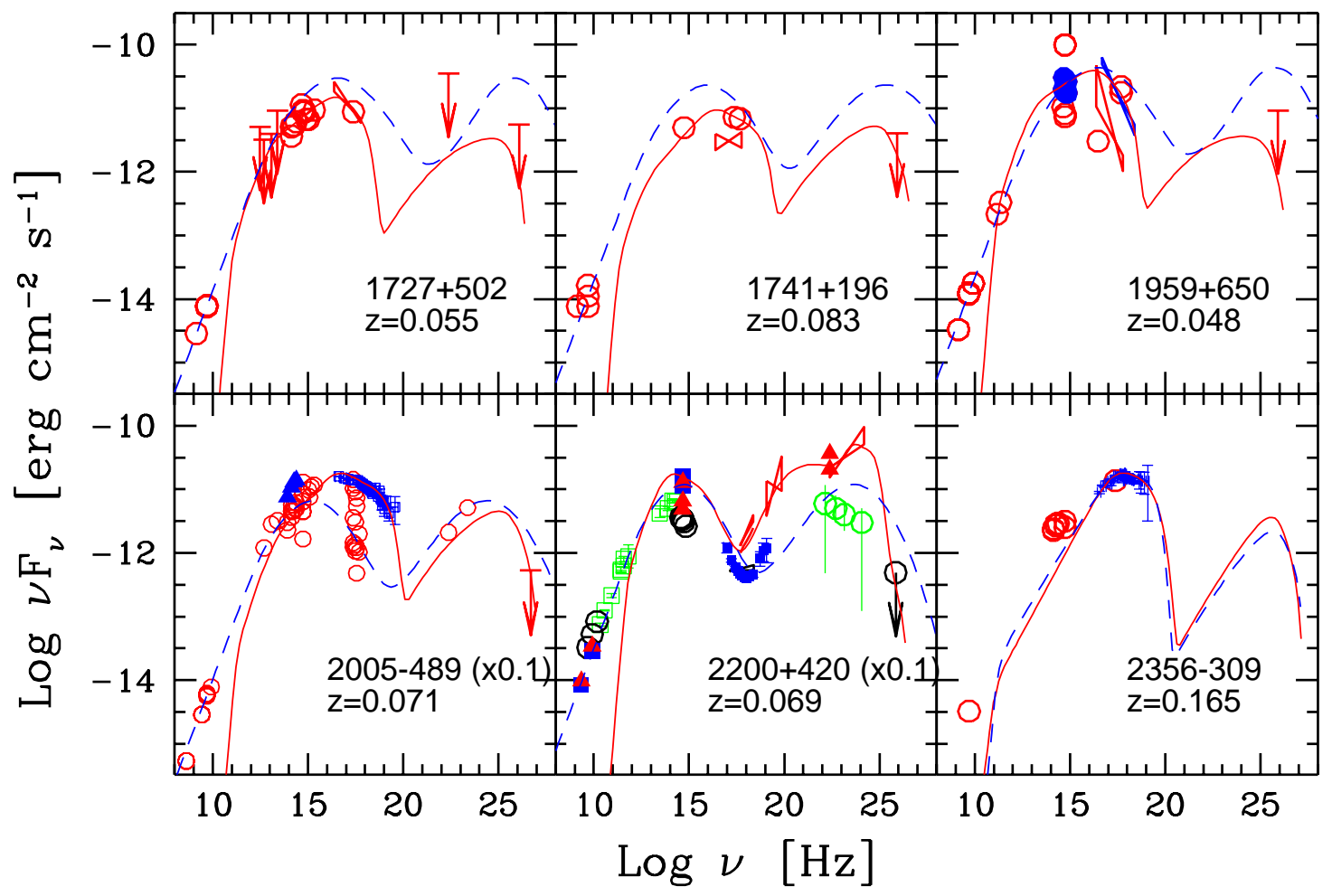

Fig. 6. d) SEDs of our best candidates for TeV emission. The solid lines refer to the SSC model as explained in Sect. 5.1. Dashed lines correspond to the phenomenological prescription of Fossati et al. (1998), as slightly modified by Donato et al. (2001) and in this paper. Sources of data listed in Table 2.

study their intrinsic spectrum in an unabsorbed band. In addition this energy range will link ground based Cherenkov observations and the data coming from satellites, such as AGILE and GLAST, observing from a few tens of $\mathrm{MeV}$ to a few tens of $\mathrm{GeV}$.

As a final note, we warn that our flux estimates do not include the possible absorption due to the infrared background, since we preferred to be independent of this factor. In fact, we focussed on the conditions for the TeV emission, providing a list of possible sources, in order to allow an independent test of the IR absorption effects. This differs with respect to the flux estimates in Stecker et al. (1996), which instead account for the IR background absorption. However, since most of the photon flux is expected to be emitted at energies below $1 \mathrm{TeV}$ (as can be seen in Table 3, comparing the values above 0.3 and $1 \mathrm{TeV}$ ), the reported fluxes should not be much affected for sources up to $z \sim 0.1$, according to the present estimates on the IR background (see Stecker 2001 and references therein).

Acknowledgements. We thank the referee, H. Krawczynski, for his constructive suggestions. We thank D. A. Smith, A. Djannati-Atai, I. de la Calle Perez, F. Piron and A. Celotti for useful discussions. This research has made use of the NASA/IPAC Extragalactic Database (NED) which is operated by the Jet Propulsion Laboratory, Caltech, under contract with the National Aeronautics and Space Administration.

\section{References}

Aharonian, F. A., Akhperjanian, A. G., Barrio, J. A., et al. 1997, A\&A, 327, L5

Aharonian, F. A., Akhperjanian, A. G., Barrio, J. A., et al. 2000, A\&A, 353, 847

Aharonian, F. A. 2000, New Astron. 5, 377 [astro-ph/0003159]

Aharonian, F. A., Timokhin, A. N., \& Plyasheshnikov, A. V. 2001 [astro-ph/0108419]

Aharonian, F. A. 2001b, 27th International Cosmic ray Conference, Hamburg, Germany, in press

Amelino-Camelia, G., \& Piran, T. 2001, Phys. Rev., D64, 036005 [astro-ph/0008107]

Bade, N., Fink, H., \& Engels, D. 1994, A\&A, 286, 381

Bade, N., Beckmann, V., Douglas, N. G., et al. 1998, A\&A, 334,459

Bauer, F. E., Condon, J. J., Thuan, T. X., \& Broderick, J. J. 2000, ApJS, 129, 547

Beckmann, V., Wolter, A., Celotti, A., et al. 2001, A\&A, in press

Berezinsky, V. 2001, 9th Int. Workshop Neutrino Telescopes [astro-ph/0107306]

Bersanelli, M., Bouchet, P., Falomo, R., \& Tanzi, E. G. 1992, AJ, 104, 28

Brinkmann, W., \& Siebert, J. 1994, A\&A, 285, 812

Brinkmann, W., Siebert, J., Reich, W., et al. 1995, A\&AS, 109, 147

Brinkmann, W., Yuan, W., \& Siebert, J. 1997, A\&A, 319, 413

Brown, L. M., Robson, E. I., Gear, W. K., et al. 1989, ApJ, 340,129 
Buckley, J. H. 1999, APh, 11, 119

Burstein, D., \& Heiles, C. 1982, AJ, 87, 1165

Catanese, M., Akerlof, C. W., Biller, S. D., et al. 1997a, ApJ, 480,562

Catanese, M., Bradbury, S. M., Breslin, A. C., et al. 1997b, ApJ, 487, L147

Catanese, M., \& Weekes, T. C. 1999, PASP, 111, 1193

Celotti, A. 2001, Blazar Physics and Demographics, ed. M. C. Urry, \& P. Padovani, ASP, 227, 105

Chadwick, P. M., Lyons, K., MCComb, T. J. L., et al. 1999, ApJ, 521, 547

Ciliegi, P., Bassani, L., \& Caroli, E. 1995, ApJ, 439, 80

Comastri, A., Fossati, G., Ghisellini, G., \& Molendi, S. 1997, ApJ, 480, 534

Costamante, L., Ghisellini, G., Tagliaferri, G., et al. 2001a, Stellar Endpoints, AGN and the Diffuse Background, in press [astro-ph/0001410]

Costamante, L., Ghisellini, G., Giommi, P., et al. 2001b, A\&A, 371,512

Dermer, C., Schlickeiser, R., \& Mastichiadis, A. 1992, A\&A, 256, L27

Dingus, B. L., Bertsch, D. L., Digel, S. W., et al. 1996, ApJ, 467,589

Djannati-Ataï, A., Piron, F., Barrau, A., et al. 1999, A\&A, 350,17

Donato, D., Ghisellini, G., Tagliaferri, G., \& Fossati, G. 2001, A\&A, 375, 739

Edelson, R. A. 1994, AJ, 94, 1150

Fabian, A. C., Celotti, A., Iwasawa, K., et al. 2001a, MNRAS, 323,373

Fabian, A. C., Celotti, A., Iwasawa, K., \& Ghisellini, G. 2001b, MNRAS, 324, 628

Falomo, R., \& Treves, A. 1990, PASP, 102, 1120

Falomo, R., Scarpa, R., \& Bersanelli, M. 1994, ApJS, 93, 125

Fichtel, C. E., Bertsch, D. L., Chiang, J., et al. 1994, ApJS, 94,551

Fossati, G., Celotti, A., Ghisellini, G., \& Maraschi, L. 1997, MNRAS, 289, 136

Fossati, G., Maraschi, L., Celotti, A., Comastri, A., \& Ghisellini, G. 1998, MNRAS, 299, 433 (F98)

Fossati, G., Celotti, A., \& Chiaberge, M. 2000, ApJ, 541, 153

Fruscione, A. 1996, ApJ, 459, 509

Gaidos, J. A., Akerlof, C. W., Biller, S. D., et al. 1996, Nature, 383,319

Gear, W. K., Stevens, J. A., Hughes, D. H., et al. 1994, MNRAS, 267, 167

George, I. M., \& Turner, T. J. 1996, ApJ, 461, 198

Ghisellini, G., \& Madau, P. 1996, MNRAS, 280, 67

Ghisellini, G., Celotti, A., Fossati, G., Maraschi, L., \& Comastri, A. 1998, MNRAS, 301, 451

Ghisellini, G. 1999, The Active X-ray Sky: Results from BeppoSAX and Rossi-XTE, Nucl. Physics B Proc. Supp., ed. L. Scarsi, H. Bradt, P. Giommi, \& F. Fiore, 397

Ghisellini, G., Celotti, A., \& Costamante, L. 2001, A\&A, submitted

Ghosh, K., \& Soundararajaperumal, S. 1995, ApJS, 100, 37

Giommi, P., Ansari, S. G., \& Micol, A. 1995, A\&AS, 109, 267

Giommi, P., Barr, P., Garilli, B., Maccagni, D., \& Pollock, A. M. T. 1990, ApJ, 356, 432

Giommi, P., Padovani, P., \& Perlman, E. 1997, MNRAS, 317, 743

Hartman, R. C., Bertsch, D. L., Bloom, S. D., et al. 1999, ApJS, 123, 79
Hauser, G. H., \& Dwek, E. 2001, ARA\&A, 39 [astro-ph/0105539]

Horan, D., VERITAS collaboration, 2000, HEAD meeting, No. 32, 05.03

Horan, D., Badran, H. M., Bond, I. H., et al. 2001, AIP Conf. Proc., 578, Gamma-Ray Astrophys. 2001, ed. S. Ritz, N. Gehrels, \& C. R. Schrader, 324

Inpey, C. D., \& Neugebauer, G. 1988, AJ, 95, 307

Kerrick, A. D., Akerlof, C. W., Biller, S., et al. 1995, ApJ, 452, 588

Kifune, T., Dazeley, S. A., Edwards, P. G., et al. 1997 [astro-ph/9707001]

Krawczynski, H., Sambruna, R., Kohnle, A., et al. 2001, ApJ, 559, 187

Krennrich, F., Badran, H. M., Bond, I. H., et al. 2001, ApJ, 560,45

Lamer, G., Brunner, H., \& Staubert, R. 1996, A\&A, 311, 384

Laurent-Muehleisen, S. A., Kollgaard, R. I., Feigelson, E. D., Brinkmann, W., \& Siebert, J. 1999, ApJ, 525, 127

Litchfield, S. J., Robson, E. I., \& Stevens, J. A. 1994, MNRAS, 270,341

Macomb, D. J., Akerlof, C. W., Aller, H. D., et al. 1995, ApJ, 449, 99

Madejski, G. M., \& Schwartz, D. A. 1988, ApJ, 330, 776

Madejski, G. M., Sikora, M., Jaffe, T., et al. 1999, ApJ, 521, 145

Mannheim, K. 1993, A\&A, 269, 67

Maraschi, L., Fossati, G., Tavecchio, F., et al. 1999, ApJ, 526,81

Muecke, A., \& Protheroe, R. J. 2000 [astro-ph/0004052]

Neshpor, Y. I., Stepanyan, A. A., Kalekin, O. P., et al. 1998, Astron. Lett., 24, 134

Neumann, M., Reich, W., Fuerst, E., et al. 1994, A\&AS, 106, 303

Nishiyama, T., Chamoto, N., Chikawa, M., et al. 1999, in Proc. of the 26th ICRC, ed. D. Kieda et al., 3, 370

Padovani, P., \& Giommi, P. 1995, ApJ, 444, 567

Padovani, P., Costamante, L., Giommi, P., et al. 2001, MNRAS, in press

Perlman, E. S., Stocke, J. T., Schacter, J. F., et al. 1996, ApJS, 104,251

Pian, E., \& Treves, A. 1993, ApJ, 416, 130

Pian, E., Vacanti, G., Tagliaferri, G., et al. 1998, ApJ, 492, 17

Piran, T. 1999, Phys. Rep., 314, 575

Piron, F. 2000, Ph.D. Thesis, University of Paris-Sud, Paris XI

Protheroe, R. J., et al. 1998, 25th International Cosmic Ray Conf., ed. M. S. Potgieter, B. C. Raubenheimer, \& D. J. van der Walt (Singapore River Edge, NJ, World Scientific), 317 [astro-ph/9710118]

Protheroe, R. J., \& Meyer, H. 2000, Phys. Lett. B, 493, 1

Punch, M., Akerlof, C. W., Cawley, M. F., et al. 1992, Nature, 358, 477

Rachen, J. P. 1999, GeV-TeV Gamma-Ray Astrophysics Workshop (Snowbird, USA), 41

Rector, T., Stocke, J. T., Perlman, E. S., Morris, S. L., \& Gioia, I. 2000, AJ, 120, 1626

Roberts, M. D., McGee, P., \& Dazeley, S. A. 1999 [astro-ph/9902008]

Robson, E. I., Stevens, J. A., \& Jenness, T. 2001, MNRAS, in press [astro-ph/0107112]

Sambruna, R. M., Barr, P., Giommi, P., et al. 1994, ApJS, 95, 371

Sambruna, R. M., Ghisellini, G., Hooper, E., et al. 1999, ApJ, 515,140 
Sikora, M., Begelman, M. C., \& Rees, M. J. 1994, ApJ, 421, 153

Sitko, M. L., \& Sitko, A. K. 1991, PASP, 103, 160

Spada, M., Ghisellini, G., Lazzati, D., \& Celotti, A. 2001, MNRAS, 325, 1559

Stecker, F. W., De Jager, O. C., \& Salamon, M. H. 1992, ApJ, 390, 49

Stecker, F. W., De Jager, O. C., \& Salamon, M. H. 1996, ApJ, 473, 75

Stecker, F. W., \& De Jager, O. C. 1998, A\&A, 334, L85

Stecker, F. W. 2001, Proc. IAU Symp. 204, The Extragalactic Background and its Cosmological Implications, ed. M. Harwit, \& M. G. Hauser [astro-ph/0010015]

Stevens, J. A., Litcfield, S. J., Robson, E. I., et al. 1994, ApJ, 437,91

Stevens, J. A., \& Gear, W. K. 1999, MNRAS, 307, 403

Stickel, M., Fried, J. W., \& Kuhr, H. 1993, A\&AS, 98, 393

Tagliaferri, G., Ghisellini, G., Giommi, P., et al. 2001, A\&A, 368,38
Takahashi, T., Kataoka, J., Madejski, G., et al. 2000, ApJ, 542, 105

Tavecchio, F., Maraschi, L., \& Ghisellini, G. 1998, ApJ, 509, 608

Teraesranta, H., Tornikoski, M., Mujunen, A., et al. 1996, A\&AS, 116, 157

Urry, C. M., Sambruna, R. M., Worrall, D. M., et al. 1996, ApJ, 463, 424

Weekes, T. 2000, Proc. of GeV-TeV Gamma Ray Astrophys. Workshop (Snowbird), ed. B. L. Dingus, M. H. Salamon, \& D. B. Kieda, 515, 3 [astro-ph/9910394]

Wolter, A., Comastri, A., Ghisellini, G., et al. 1998, A\&A, 335, 899

Wolter, A., Tavecchio, F., Caccianiga, A., Ghisellini, G., \& Tagliaferri, G. 2000, A\&A, 357, 429

Worrall, D. M., \& Wilkes, B. J. 1990, ApJ, 360, 396 